TRANSACTIONS OF THE

AMERICAN MATHEMATICAL SOCIETY

Volume 359, Number 2, February 2007, Pages 789-825

S 0002-9947(06)03920-1

Article electronically published on September 11, 2006

\title{
TANGENTIALLY POSITIVE ISOMETRIC ACTIONS AND CONJUGATE POINTS
}

\author{
RAÚL M. AGUILAR
}

\begin{abstract}
Let $(\mathrm{M}, g)$ be a complete Riemannian manifold with no conjugate points and $f:(\mathrm{M}, g) \rightarrow\left(\mathrm{B}, g_{\mathrm{B}}\right)$ a principal $G$-bundle, where $G$ is a Lie group acting by isometries and B the smooth quotient with $g_{\mathrm{B}}$ the Riemannian submersion metric.

We obtain a characterization of conjugate point-free quotients $\left(\mathrm{B}, g_{\mathrm{B}}\right)$ in terms of symplectic reduction and a canonical pseudo-Riemannian metric on the tangent bundle $T \mathrm{M}$, from which we then derive necessary conditions, involving $G$ and $\mathrm{M}$, for the quotient metric to be conjugate point-free, particularly for $\mathrm{M}$ a reducible Riemannian manifold.

Let $\mu_{G}: T \mathrm{M} \rightarrow \mathfrak{G}^{*}$, with $\mathfrak{G}$ the Lie Algebra of $G$, be the moment map of the tangential $G$-action on $T \mathrm{M}$ and let $\mathbf{G}_{\mathbf{P}}$ be the canonical pseudo-Riemannian metric on $T \mathrm{M}$ defined by the symplectic form $d \Theta$ and the map $F: T \mathrm{M} \rightarrow$ $\mathrm{M} \times \mathrm{M}, F(z)=(\exp (-z), \exp (z))$. First we prove a theorem, stating that if $\mathbf{G}_{\mathbf{P}}$ is not positive definite on the action vector fields for the tangential action along $\mu_{G}{ }^{-1}(0)$ then $\left(\mathrm{B}, g_{\mathrm{B}}\right)$ acquires conjugate points. (We proved the converse result in 2005.) Then, we characterize self-parallel vector fields on $\mathrm{M}$ in terms of the positivity of the $\mathbf{G}_{\mathbf{P}}$-length of their tangential lifts along certain canonical subsets of $T \mathrm{M}$. We use this to derive some necessary conditions, on $G$ and M, for actions to be tangentially positive on relevant subsets of $T \mathrm{M}$, which we then apply to isometric actions on complete conjugate point-free reducible Riemannian manifolds when one of the irreducible factors satisfies certain curvature conditions.
\end{abstract}

\section{INTRODUCTION}

Let $(\mathrm{M}, g)$ be a connected, smooth manifold of dimension $n$ with a complete Riemannian metric with no conjugate points. (M has conjugate points $p$ and $q$ if the differential of the exponential map defined by $g$, $\exp _{*}: T_{p} \mathrm{M} \rightarrow T_{q} \mathrm{M}$, has non-trivial kernel; e.g., antipodal points when $\mathrm{M}$ is an Euclidean sphere. See 11 and 12 for basic definitions.) Let $G$ be a Lie group, acting isometrically, properly, and freely on $\mathrm{M}$ with a smooth manifold $\mathrm{B}=\mathrm{M} / G$ as its quotient. We give $\mathrm{B}$ the unique Riemannian metric $g_{\mathrm{B}}$ so that the principal $G$-bundle

$$
f:(\mathrm{M}, g) \rightarrow\left(\mathrm{B}, g_{\mathrm{B}}\right)
$$

is a also Riemannian submersion, defined by $g_{\mathrm{B}}\left(f_{*} u, f_{*} v\right)=g(u, v)$ for $u$ and $v$ $g$-orthogonal to the fibers. We refer to (1.1) as a Riemannian principal $G$-bundle. It is well known that the metric $g_{\mathrm{B}}$ is complete $\left(\left[\begin{array}{l}7 \\ )\end{array}\right)\right.$.

Received by the editors January 8, 2004 and, in revised form, December 16, 2004.

2000 Mathematics Subject Classification. Primary 53C20, 53C22, 53D20, 53D25.

Key words and phrases. Moment map, isometric action, conjugate points, symplectic reduction. 
In the particular case when $\operatorname{dim} G=0$, known as a Riemannian cover, $\left(\mathrm{B}, g_{\mathrm{B}}\right)$ is locally isometric to the total space via the projection $f$. Thus, many metric properties of $(\mathrm{M}, g)$, including the non-existence of conjugate points, are inherited by $\left(\mathrm{B}, g_{\mathrm{B}}\right)$. But, in general, a submersion with fibers of positive dimension, such as (1.1) when $\operatorname{dim} G>0$, is curvature non-decreasing, and so is the possibility of the existence of conjugate points in the base.

In this article, a continuation of [1, we use the symplectic reduction procedure in the tangent bundle and a canonical product structure there to address the generation of conjugate points by quotients of isometric actions by Lie groups of positive dimension. This approach should be viewed as a 'Pseudo-Riemannian reduction' and compared to the Kähler reduction of the so-called 'adapted complex structure' of Lempert-Szöke [13] and Guillemin-Stenzel [6] that we carried out in [2]. The main difference with the Kähler case, as already shown in [1, is that due to the non-definiteness of the metric involved, the Pseudo-Riemannian reduction away from the zero section of TM might fail. (See Section 7) However, as it follows from [1] and this present work, this failure occurs precisely when conjugate points are created in the quotient as we now outline, and this allows several applications.

Via the induced tangential $G$-action on the tangent bundle $T \mathrm{M}$ (the action generated by the tangent maps of the flow of the original $G$-action on M) $G$ acts on $\mu_{G}{ }^{-1}(0) \subset T \mathrm{M}$, where

$$
\mu_{G}: T \mathrm{M} \rightarrow \mathfrak{G}^{*},
$$

with $\mathfrak{G}^{*}$ the dual of the Lie algebra $\mathfrak{G}$ of $G$, is the moment map of the tangential $G_{0}$-action, where $G_{0} \subset G$ is the connected component containing the identity element. The assumption that $(\mathrm{M}, g)$ has no conjugate points implies that the pseudo-Riemannian metric $\mathbf{G}_{\mathbf{P}}$ of signature $(n, n)$ defined for $U$ and $V$ in $T(T \mathrm{M})$ as

$$
\mathbf{G}_{\mathbf{P}}(U, V)=d \Theta(\mathbf{P} U, V)
$$

is defined in all of $T \mathrm{M}$. Here $d \Theta$ is the canonical symplectic form on $T \mathrm{M}$ induced by the metric $g$ as in (2.1), and $\mathbf{P}$ is the structure induced by the map $F: T \mathrm{M} \rightarrow \mathrm{M} \times \mathrm{M}$ given by $F(z)=(\exp (-z), \exp (z))$. (See Section 2,

We need to recall the following definition introduced in [1].

Definition 1.1. An isometric $G$-action on $(\mathrm{M}, g)$ is tangentially positive on a set $\mathcal{S} \subset T \mathrm{M}$ iff for every action vector field $\xi_{T \mathrm{M}}$ on $T \mathrm{M}, \xi \in \mathfrak{G}$, it holds $\left\|\xi_{T \mathrm{M}}(z)\right\|_{\mathbf{G}_{\mathrm{P}}}^{2}>0$ at every $z \in \mathcal{S}$ where $\xi_{T \mathrm{M}}(z) \neq 0$.

(In Definition 1.1 the $G$-action is not assumed free. In fact, a trivial action is automatically tangentially positive on all $T$ M. Similarly if $\operatorname{dim} G=0$.)

By Theorem 3 of 1 if the $G$-action defining the Riemannian principal bundle $f:(\mathrm{M}, g) \rightarrow\left(\mathrm{B}, g_{\mathrm{B}}\right)$ is tangentially positive on $\mu_{G}^{-1}(0) \subset T \mathrm{M}$, then $\mathrm{B}$ has no conjugate points.

In this paper we show the converse of the above-mentioned result from [1, namely

Theorem 1.2. Let both the total space and the base of the Riemannian principal G-bundle,

$$
f:(\mathrm{M}, g) \rightarrow\left(\mathrm{B}, g_{B}\right),
$$

have no conjugate points. Then $G$ acts tangentially positively on $\mu_{G}{ }^{-1}(0)$. 
Collecting Theorem 1.2 in this paper and Theorem 3 in [1] we have a proof of the following.

Theorem. Let the total space $(\mathrm{M}, g)$ of (1.4) have no conjugate points. Then $\left(\mathrm{B}, g_{B}\right)$ has no conjugate points if and only if $G$ acts tangentially positively on $\mu_{G}{ }^{-1}(0)$.

We exploit the result above to derive some restrictions on the existence of isometric actions on conjugate point-free manifolds with conjugate point-free quotients. As a sample we state now as a theorem what is later stated and proved as Corollary 6.11 of Theorem 6.10.

Theorem (*). Let $\left(\mathrm{M}=\mathrm{M}_{1} \times \mathrm{M}_{2}, g_{1} \times g_{2}\right)$ be a complete Riemannian product with no conjugate points, where $\left(\mathrm{M}_{1}, g_{1}\right)$ is non-positively curved of rank one, or has negative-definite Ricci tensor at some point $p$. Let $G$ act properly isometrically on $\mathrm{M}$ preserving the splitting, and freely on $\mathrm{M}_{2}$. If the quotient $\mathrm{B}=\mathrm{M}_{1} \times_{G} \mathrm{M}_{2}$ with the submersion metric has no conjugate points, then $\mathrm{B}$ is a (possibly trivial) product with a factor that is locally isometric to $\mathrm{M}_{1}$.

A particular case of Theorem $(*)$ is when $G$ acts diagonally on $\left(\mathrm{M}=\mathrm{M}_{1} \times \mathrm{M}_{1}\right.$, $\left.g_{1} \times g_{1}\right)$, that is, $h \cdot(x, y)=(h \cdot x . h \cdot y)$ for all $h \in G$, freely and properly so that the smooth quotient $\mathrm{M}_{1} \times{ }_{G} \mathrm{M}_{1}$ has no conjugate points. Our result implies that, with $\left(\mathrm{M}_{1}, g_{1}\right)$ as above, this can only happen if $\operatorname{dim} G=0$.

Another case covered by Theorem $\left(^{*}\right)$ is when we attempt to 'deform' the metric in $\mathrm{M}_{1}$ by taking the Riemannian quotient $\mathrm{B}=\mathrm{M}_{1} \times_{G} G$ for $G$ a connected nonpositively curved Lie group acting isometrically on itself by group multiplication and isometrically on $\mathrm{M}_{1}$. Then, when $\mathrm{M}_{1}$ is as in Theorem $\left(^{*}\right)$, B is isometric to $\mathrm{M}_{1}$ or has conjugate points.

Note that no compactness assumption is made here for $\mathrm{M}_{1}$, so the above does not follow from the classical theorem of S. Bochner [3] (which would actually require, in addition to compactness, negative-definiteness for the Ricci tensor on all of $\mathrm{M}_{1}$, not just a point).

The statement of Theorem $\left(^{*}\right)$ is typical of the results on conjugate points we obtain here in that it refers to Riemannian products with an assumption made on the rank or Ricci curvature at one point in one of the factors. This is because our results are derived in part from Theorem 4.1, by which we characterize, for any given vector field $X: \mathrm{M} \rightarrow T \mathrm{M}$, the positivity of the $\mathbf{G}_{\mathbf{P}^{-}}$-length of its tangential lift along the set $\mathcal{S}_{X} \stackrel{\text { def }}{=}\{\mathbb{R} X(p) \mid p \in \mathrm{M}\} \subset T \mathrm{M}$ in terms of the 'self-parallelism' of $X$, $\nabla_{X} X=0$. But $\mu_{G}^{-1}(0) \cap \mathcal{S}_{X}=\mathrm{M}$, here $\mathrm{M}$ viewed as the zero section of $T \mathrm{M}$, and Theorem 1.2 and Theorem 4.1 refer to different subsets of $T \mathrm{M}$. However, we take advantage of both theorems in the context of reducible Riemannian manifolds and splitting-preserving actions by working with non-action vector fields $X=X_{1}+X_{2}$ on the product such that $\mathcal{S}_{X} \subset \mu_{G}{ }^{-1}(0)$, and whose projection to one of the factors, say $\mathrm{M}_{1}$, is an action vector field for the induced action on $\mathrm{M}_{1}$. Then, the self-parallelism of such projection $X_{1}$ imposes curvature restrictions on $\mathrm{M}_{1}$.

The role of $\mathbf{P}$ in proving Theorem 1.2 (and Theorem 3 in [1]) is as follows. First, the structure $\mathbf{P}$ can be characterized symplectically, on a certain kind of set that we call a serrate set, contained in neighborhoods of the zero section, as the unique integrable local product structure with the property that $\mathbf{P} d E=\Theta$, where $E$ is the energy function in (2.1); furthermore, its existence on the whole $T \mathrm{M}$ is equivalent to the absence of conjugate points (see Section 2). Second, by the classical symplectic 
reduction procedure, given the isometric $G$-action one identifies $\mu_{G}{ }^{-1}(0) / G$ with $T$ B symplectically, that is, the induced pushed-down one-form $\check{\Theta}$ and function $\check{E}$ agree on $T \mathrm{~B}$ with the canonical objects $\Theta_{\mathrm{B}}$ and $E_{\mathrm{B}}$ on $T \mathrm{~B}$ defined by the submersion metric $g_{\mathrm{B}}$. But this classical reduction construction links to the uniqueness of $\mathbf{P}$, giving the proof we seek, as explained next.

In one direction, as long as the action remains tangentially positive on $\mu_{G}{ }^{-1}(0)$, the pseudo-Riemannian metric $\mathbf{G}_{\mathbf{P}}$ defines a connection for the principal $G$-bundle $\mu_{G}^{-1}(0) \rightarrow T$ B with $\mathbf{P}$-invariant horizontal distribution. (So, $\mathbf{G}_{\mathbf{P}}$ is a pseudoRiemannian extension of $g$; it also extends the $G$-connection defined by $g$, since the horizontal distribution on $\mu_{G}{ }^{-1}(0)$ is defined by the $\mathbf{G}_{\mathbf{P}}$-orthogonal complement to the fibers.) Thus, we can push-down $\mathbf{P}$ to a $\check{\mathbf{P}}$ defined on all $T \mathrm{~B}$ and satisfying the characterizing properties of the canonical $\mathbf{P}_{\mathrm{B}}$, hence equal to $\mathbf{P}_{\mathrm{B}}$ by the uniqueness result on serrate sets mentioned above, forcing $\left(\mathrm{B}, g_{\mathrm{B}}\right)$ to be conjugate point-free.

On the other direction, if the action fails to be tangentially positive on the entire $\mu_{G}{ }^{-1}(0)$, there is a point $z^{c} \in \mu_{G}{ }^{-1}(0)$ where the action is not tangentially positive, so that it can be approached as closely as we want by the top vertices of certain 'triangular' two-dimensional serrate subsets of $\mu_{G}{ }^{-1}(0)$ where the connection is defined by $\mathbf{G}_{\mathbf{P}}$. At the $f_{*}$-image of these serrate sets the pushed-down $\check{\mathbf{P}}=P_{\mathrm{B}}$, as endomorphisms of $T \mathrm{~B}$. But at $z^{c}$ there is a vector $U$ in $T_{z^{c}}\left(\mu_{G}{ }^{-1}(0)\right)$, both transversal and $\mathbf{G}_{\mathbf{P}}$-orthogonal at $z^{c}$ to the orbits of the $G$-action on $\mu_{G}{ }^{-1}(0)$, and a limiting argument shows that the (non-zero) image of $U$ by $\left(f_{*}\right)_{*}$ must be in the kernel of $\mathbf{P}_{\mathrm{B}}$, forcing $\left(\mathrm{B}, g_{\mathrm{B}}\right)$ to have conjugate points.

The rest of the paper is organized as follows.

Section 2: preliminaries; proof of uniqueness of the structure $\mathbf{P}$ on serrate sets (a necessary refinement of what we have proved in [1]).

Section 3. proof of Theorem 1.2 stated in the Introduction; set up, proofs, and review of the proof of the naturality of $\mathbf{P}$ under symplectic reduction obtained in [1.

Section 44: G $\mathbf{P}_{\mathbf{P}}$-length of tangential lifts; statement of Theorem 4.1] $G_{\mathbf{P}}$ along the Riemannian leaves defined by the geodesics; proof of Theorem 4.1 .

Section 5 , propositions on tangential positivity; restrictions on the curvature of the manifold acted upon and on the structure of the fixed-point set when the $G$-action is tangentially positive in two canonical subsets of $T \mathrm{M}$, one of which is $\mu_{G}^{-1}(0)$.

Section 6. Theorem 6.1 characterizing tangential positivity on $\mu_{G}^{-1}(0)$ for Euclidean $\mathbb{R}^{n}$; Theorems 6.5 and 6.10 for Riemannian products.

Section 7 examples referred to in previous sections; remarks on the reduction of $\mathrm{P}$ versus the one for the "adapted complex structure".

\section{Preliminaries}

Let $(\mathrm{M}, g)$ be a connected, smooth and complete Riemannian manifold of dimension $n$ and $\pi: T \mathrm{M} \rightarrow \mathrm{M}$ its tangent bundle. We recall that complete means that every geodesic in $\mathrm{M}$ is defined for the arc-length parameter in $(-\infty, \infty)$. M will not be assumed compact unless said so. The metric $g$ defines the energy function $E: T \mathrm{M} \rightarrow \mathbb{R}$ and the one-form $\Theta$ on $T \mathrm{M}$ so that for all $z \in T \mathrm{M}, U \in T_{z}(T \mathrm{M})$

$$
E(z)=\frac{1}{2}\|z\|^{2}=\frac{1}{2} g(z, z), \quad \Theta(U)=g\left(\pi_{*} U, z\right) .
$$


Consider the endomorphism $\mathbf{P}$ of $\left.T(T \mathrm{M})\right|_{\mathcal{O}}$, with $\mathcal{O} \subset T \mathrm{M}$ a neighborhood of the zero section $\mathrm{M}$, defined by the map $F: T \mathrm{M} \rightarrow \mathrm{M} \times \mathrm{M}$ given by $F(z)=$ $(\exp (-z), \exp (z))$, where $\exp : T \mathrm{M} \rightarrow \mathrm{M}$ is the standard exponential map that sends $z \in T_{p} \mathrm{M}$ to $\gamma(\|z\|) \in \mathrm{M}, \gamma$ being the unit-speed geodesic such that $\|z\| \dot{\gamma}(0)=z$ if $z \neq 0$, or to $p$ if $z=0 \in T_{p} \mathrm{M}$. Here $\mathcal{O}$ is the open set where $F$ is a local diffeomorphism, hence $\mathcal{O}=T \mathrm{M}$ if and only if $(\mathrm{M}, g)$ have no conjugate points. $\mathbf{P}$ is the pull back of the endomorphism in $T(\mathrm{M} \times \mathrm{M})$ that acts as $-\mathbf{I}$ on the tangent spaces of the left M-factor and as $\mathbf{I}$ on the ones of the right M-factor. Such $\mathbf{P}$ satisfies

$$
\left\{\begin{array}{l}
\mathbf{P}^{2}=\mathbf{I} \text { (= identity endomorphism); } \\
\mathbf{P} \text { is integrable; } \\
\mathbf{P} d E=\Theta \text { (i.e., } \forall U, d E(\mathbf{P} U)=\Theta(U)) .
\end{array}\right.
$$

Integrability of $\mathbf{P}$ means that there are local charts $\left\{u_{1}^{-}, \cdots, u_{n_{-}}^{-}, u_{1}^{+}, \cdots, u_{n_{+}}^{+}\right\}$of $\mathcal{O}$, with $n_{+}+n_{+}=n$, such that $\mathbf{P}\left(\frac{\partial}{\partial u_{i}^{ \pm}}\right)= \pm \frac{\partial}{\partial u_{i}^{ \pm}}$. Of course, $n_{-}=n_{+}=n$ in this case. We call such coordinates, product coordinates for $\mathbf{P}$. The first two properties are obvious, while the third requires some checking and is proved in [1]. That $\mathcal{O}$ contains the zero section $M$ follows in a standard way.

In [1] we called any structure $\mathbf{P}^{\prime}$ that satisfies the three conditions (2.2), in principle different from $\mathbf{P}$, an adapted product structure (see remarks in subsection 3.1.1). We need a slight refinement of the uniqueness result for adapted product structures, "P' $=\mathbf{P}$ on certain open sets", proven in Theorem 1 of [1].

First we give a definition of a serrate set different but equivalent to the one given in [1, and more useful for us now. Given a non-trivial geodesic $\gamma: \mathbb{R} \rightarrow \mathrm{M}$ parametrized by arc-length, we identify $T \mathbb{R} \cong \mathbb{R}^{2}$ so that $\gamma_{*}(x, y)=y \dot{\gamma}(x)$ for all $(x, y)$ in $\mathbb{R}^{2}$. If the geodesic is trivial, that is, if $\gamma(\mathbb{R})=\{p\} \in \mathrm{M}$, then $\gamma_{*}(x, y)=0_{p}$, the zero vector in $T_{p} \mathrm{M}$ which we identify with $p$.

Definition 2.1. Given $0 \neq z \in T \mathrm{M}$ we let $\gamma_{z}$ be the unit-speed geodesic of M such that $\|z\| \dot{\gamma}_{z}(0)=z$; if $z=0_{p}$ we let $\gamma_{z}$ be the trivial geodesic with image $\{p\}$.

For $0 \leq t \in \mathbb{R}$ set

$$
\triangle_{t} \stackrel{\text { def. }}{=} \text { convex hull of }\{(-t, 0),(t, 0),(0, t)\} \subset \mathbb{R}^{2} .
$$

Definition 2.2 (Serrate Set). A set $\mathcal{S} \subset T \mathrm{M}$ is serrate if and only if $\forall z \in T \mathrm{M}$

$$
z \in \mathcal{S} \Longrightarrow \mathcal{S}_{z} \stackrel{\text { def. }}{=}\left(\gamma_{z}\right)_{*}\left(\triangle_{\|z\|}\right) \subset \mathcal{S} \text {. }
$$

To proceed we need the following bits of notation (see [5] or [9] for details).

Let $\mathbf{K}: T(T \mathrm{M}) \rightarrow T \mathrm{M}$ be the connection map. We recall for later reference that

$$
d \Theta(U, V)=g\left(\mathbf{K} U, \pi_{*} V\right)-g\left(\mathbf{K} V, \pi_{*} U\right) .
$$

Given $z$ and $u$ both in $T_{p} \mathrm{M}$ we let the horizontal lift of $u$ at $z,(u)_{z}^{h}$, and the vertical lift of $u$ at $z,(u)_{z}^{v}$, be the vectors in $T_{z}(T \mathrm{M})$ defined by the formulas

$$
\pi_{*}(u)_{z}^{h}=\mathbf{K}(u)_{z}^{v}=u, \quad \pi_{*}(u)_{z}^{v}=\mathbf{K}(u)_{z}^{h}=0 .
$$

Also, we denote by $\Xi$ the so-called Liouville vector field on $T \mathrm{M}$ and by $\Sigma$ the geodesic spray on $T \mathrm{M}$ generated by the geodesic flow, whose values at $z \in T \mathrm{M}$ in terms of lifts are given by

$$
\Xi(z)=(z)_{z}^{v}, \quad \Sigma(z)=(z)_{z}^{h}
$$


Proposition 2.3. Let $(\mathrm{M}, g)$ be a complete Riemannian manifold and $\mathcal{O} \supset \mathrm{M}$ an open set in TM where the structure $\mathbf{P}$ is defined. Let $\mathbf{P}^{\prime}$ be an endomorphism of $\left.T(T \mathrm{M})\right|_{\mathcal{O}}$ also satisfying (2.2) (that is, $\mathbf{P}^{\prime}$ is an adapted product structure). Then $\mathbf{P}=\mathbf{P}^{\prime}$ on any serrate set $\mathcal{S} \subset \mathcal{O}$.

Proof. According to Definitions 2.1 and 2.2, any serrate set $\mathcal{S} \subset T \mathrm{M}$ can be written as

$$
\mathcal{S}=\bigcup_{z \in \mathcal{S}} \mathcal{S}_{z}
$$

and so it is enough to show the agreement $\mathbf{P}^{\prime}=\mathbf{P}$ on $\mathcal{S}_{z}$, that is, as endomorphisms of $\left.T(T \mathrm{M})\right|_{\mathcal{S}_{z}}$, whenever $z \in T \mathrm{M}$ is such that $\mathcal{S}_{z} \subset \mathcal{O}$. (This is shown implicitly in the proof of Theorem 1 in [1]. However we outline a proof now, referring to [1] at times for further details, for the convenience of the reader and since the set up and notation are needed here in the proof of Theorem 4.1)

The condition $\mathbf{P}^{\prime} d E=\Theta$ is, in terms of (2.6), equivalent to $\mathbf{P}^{\prime} \Xi=\Sigma$, where $\Xi$ is the Liouville vector field on $T \mathrm{M}$. This follows from the facts that $d \Theta$ is nondegenerate and that $\Sigma$ is the Hamiltonian vector field corresponding to the energy function, as, $\forall U, d E(U)=d \Theta(U, \Sigma)$.

Now, $\Xi$ and $\Sigma$ vanish along the zero section M, but a limit argument shows, by continuity of $\mathbf{P}^{\prime}$ and of the horizontal and vertical distributions on $T \mathrm{M}$ associated to the connection map $\mathbf{K}$, that $\mathbf{P}^{\prime}$ is forced to act as (see Proposition 2.2 in [1])

$$
\mathbf{P}^{\prime}(u)_{0_{p}}^{h}=(u)_{0_{p}}^{v},
$$

where $0_{p}$ is the origin of $T_{p} \mathrm{M}$ and $u \in T_{p} \mathrm{M}$. From this we get the following. Let $\mathbb{R}^{2}$ be identified with $T \mathbb{R}$ by $\left.(x, y) \cong y \frac{\partial}{\partial x}\right|_{x}$. The corresponding adapted product structure $\mathbf{P}_{0}$ in $\mathbb{R}^{2}$ acts by $\mathbf{P}_{0}\left(\frac{\partial}{\partial y}\right)=\frac{\partial}{\partial x}$ and defines the global product coordinates of $\mathbb{R}^{2}$,

$$
s^{-}=x-y, \quad s^{+}=x+y .
$$

Then the map $\gamma_{*}:\left(\left(\gamma_{*}\right)^{-1} \mathcal{O} \subset \mathbb{R}^{2}, \mathbf{P}_{0}\right) \rightarrow\left(\mathcal{O} \subset T \mathrm{M}, \mathbf{P}^{\prime}\right)$ is product preserving,

$$
\mathbf{P}^{\prime} \circ \gamma_{*}=\gamma_{*} \circ \mathbf{P}_{0}
$$

if $\gamma: \mathbb{R} \rightarrow \mathrm{M}$ is a unit-speed geodesic. (Property (2.10) is more or less clear, at least off the zero section, from $\mathbf{P}^{\prime} \Xi=\Sigma$, and the expressions (2.6). See [1].)

Let $0 \neq z \in T_{p} \mathrm{M}$ and let $\gamma_{z}$ be the unit-speed geodesic given by Definition 2.1. We consider the corresponding Riemannian leaf $\mathcal{L}_{\gamma_{z}} \subset T \mathrm{M}$ which is

$$
\mathcal{L}_{\gamma_{z}}=\left\{y \dot{\gamma}_{z}(x), \mid(x, y) \in \mathbb{R}^{2}\right\} \subset T \mathrm{M} .
$$

If we let $[-a, a] \ni \lambda \mapsto \gamma^{\lambda}$ be a variation of $\gamma_{z}=\gamma^{0}$ through unit-speed geodesics $\gamma^{\lambda}: \mathbb{R} \rightarrow \mathrm{M}$, where $a>0$, the corresponding tangent variation $\lambda \mapsto\left(\gamma^{\lambda}\right)_{*}: T \mathbb{R} \rightarrow$ $T$ M defines a variation $\lambda \mapsto \mathcal{L}_{\gamma^{\lambda}}$ of $\mathcal{L}_{\gamma_{z}}$ by Riemannian leaves that restricts to the original geodesic variation along the zero section. The variation vector field obtained by taking the derivative of the tangent variation with respect to $\lambda$ and evaluating at $\lambda=0$ is a vector field $\tilde{J}$ along $\mathcal{L}_{\gamma_{z}}$, that is, $\tilde{J}$ is a section of the bundle $\left(\gamma_{z}^{*}\right)^{-1} T(T \mathrm{M})$ that restricts along $\gamma_{z}$ itself to a Jacobi field $J$ along $\gamma_{z}$. The following well-known expression is used in the next sections:

$$
\tilde{J}\left(z^{\prime}\right)=(J(p))_{z^{\prime}}^{h}+\left(\nabla_{z^{\prime}} J\right)_{z^{\prime}}^{v}, \quad \forall z^{\prime} \in \mathcal{L}_{\gamma_{z}} \cap T_{p} \mathrm{M} .
$$


Definition 2.4. We call $\tilde{J}$ the canonical extension to $\mathcal{L}_{\gamma_{z}}$ of the Jacobi field $J$ along $\gamma_{z}$.

By hypothesis $\mathbf{P}^{\prime}$ is defined in an open set $\mathcal{O}$ which contains $\mathrm{M}$. Thus, if we assume that the closed set $\mathcal{S}_{z}=\left(\gamma_{z}\right)_{*}\left(\triangle_{\|z\|}\right)=\left(\gamma^{0}\right)_{*}\left(\triangle_{\|z\|}\right) \subset \mathcal{O}$, there is $r \in \mathbb{R}$, $0<r \leq a$, and an open set $\mathcal{U} \subset \mathbb{R}^{2}$ such that

$$
\triangle_{\|z\|} \subset \mathcal{U}, \quad\left(\gamma^{\lambda}\right)_{*}(\mathcal{U}) \subset \mathcal{O}, \quad \forall \lambda \in[-r, r] .
$$

The product-preserving property (2.10) applied to each map $\gamma_{*}^{\lambda}: \mathcal{U} \subset \mathbb{R}^{2} \rightarrow T \mathrm{M}$ for fixed $\lambda \in[-r, r]$ implies that given a Jacobi field $J$ along $\gamma_{z}$, the projections of $\tilde{J}$ along $\left(\gamma_{z}\right)_{*}\left(\triangle_{\|z\|}\right) \subset \mathcal{L}_{\gamma_{z}} \cap \mathcal{O}$ into the \pm -eigen-bundles of $\mathbf{P}^{\prime}$,

$$
[\tilde{J}]_{\mathbf{P}^{\prime}}^{ \pm}=\frac{1}{2}\left(\tilde{J} \pm \mathbf{P}^{\prime} \tilde{J}\right)
$$

can be written locally, parametrizing $\mathcal{L}_{\gamma_{z}}$ by $\mathbb{R}^{2} \ni(x, y) \mapsto y \dot{\gamma}_{z}(x) \in \mathcal{L}_{\gamma_{z}}$, as

$$
[\tilde{J}(x, y)]_{\mathbf{P}^{\prime}}^{-}=\sum_{i=1}^{n} h_{i}^{-}(x-y) \frac{\partial}{\partial u_{i}^{-}}, \quad[\tilde{J}(x, y)]_{\mathbf{P}^{\prime}}^{+}=\sum_{i=1}^{n} h_{i}^{+}(x+y) \frac{\partial}{\partial u_{i}^{+}},
$$

where $h_{i}^{ \pm}$are one-variable functions and $\left\{u_{1}^{-} \cdots, u_{n}^{-}, u_{1}^{+}, \cdots, u_{n}^{+}\right\}$are local product coordinates for $\mathbf{P}^{\prime}$. (The fact that the number of \pm -coordinates for $\mathbf{P}^{\prime}$ equals $n$ is shown in [1. For more details on the above see especially Propositions 2.2 through 2.4 in [1].) It follows the next result.

Proposition 2.5. Let $\gamma=\gamma_{z}$ be a unit-speed geodesic, $J, \tilde{J}, \mathcal{O}$ and $\mathbf{P}^{\prime}$ as above, and let $s \in \mathbb{R}$. It holds (see figure below) that:

1) if $r_{1} \in \mathbb{R}$ is such that $\left\{(x-s) \dot{\gamma}(x), \forall x \mid s \leq x \leq r_{1}\right\} \subset \mathcal{O}$, then

$$
J(s)=0 \Longleftrightarrow[\tilde{J}(x, x-s)]_{\mathbf{P}^{\prime}}^{-}=0, \forall x \mid s \leq x \leq r_{1} .
$$

2) if $r_{2} \in \mathbb{R}$ is such that $\left\{(s-x) \dot{\gamma}(x), \forall x \mid r_{2} \leq x \leq s\right\} \subset \mathcal{O}$, then

$$
J(s)=0 \Longleftrightarrow[\tilde{J}(x, s-x)]_{\mathbf{P}^{\prime}}^{+}=0, \forall x \mid r_{2} \leq x \leq s .
$$

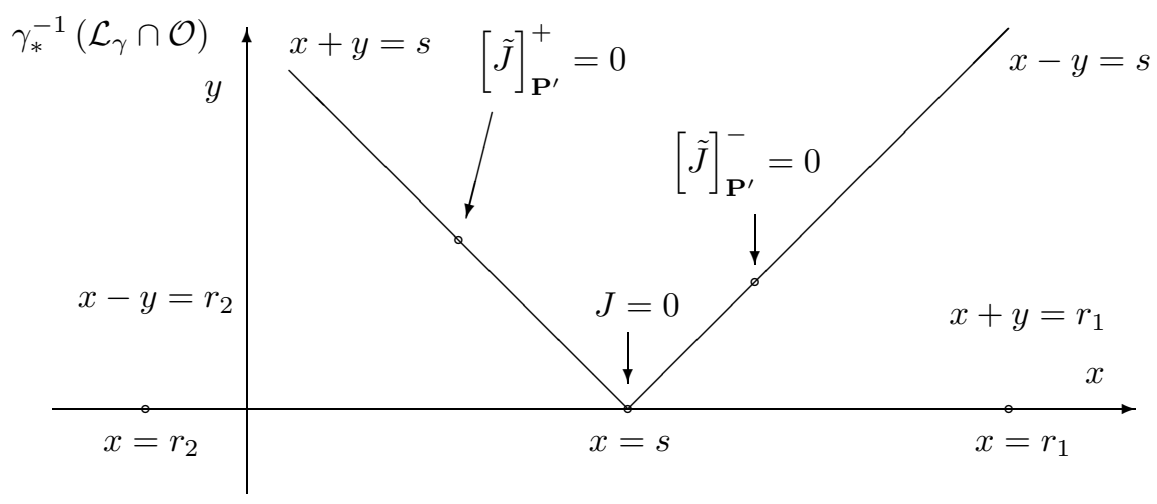

Figure For Proposition 2.5 
Proof. From the local expression (2.12) and from the fact that if $\left\{v_{i}^{ \pm}\right\}$and $\left\{u_{i}^{ \pm}\right\}$are overlapping product coordinates for $\mathbf{P}^{\prime}$, then, for all $1 \leq i, j \leq n, \frac{\partial v_{i}^{+}}{\partial u_{j}^{-}}=\frac{\partial v_{i}^{-}}{\partial u_{j}^{+}}=0$, it follows that the vanishing of $[\tilde{J}]_{\mathbf{P}^{\prime}}^{-}\left(\right.$resp. $\left.[\tilde{J}]_{\mathbf{P}^{\prime}}^{+}\right)$is a closed and open condition on the segment

$$
\left.\left\{(x, x-s) \in \mathbb{R}^{2} \mid s \leq x \leq r_{1}\right\} \text { (resp. }\left\{(x, s-x) \in \mathbb{R}^{2} \mid r_{2} \leq x \leq s\right\}\right) .
$$

On the other hand (2.8) implies $J(s)=0$ if and only if $[\tilde{J}(s)]_{\mathbf{P}^{\prime}}^{ \pm}=0$. (See [1] for more details.)

Now, assume $z \in \mathcal{S} \subset \mathcal{O}$. Since by our choice of $\gamma,\|z\| \dot{\gamma}(0)=z$, then, because $\mathcal{S}$ is serrate

$$
\gamma_{*}\left(\triangle_{\|z\|}\right) \subset \mathcal{S} \cap \mathcal{L}_{\gamma} \subset \mathcal{O} \cap \mathcal{L}_{\gamma}
$$

But Proposition 2.5 can be applied to every segment contained in $\triangle_{\|z\|} \subset \mathbb{R}^{2}$ that intersects the base of $\triangle_{\|z\|}$ at an angle $\pi / 4$ or $3 \pi / 4$. From this we conclude that:

a) the geodesic segment $\{\gamma(t) \mid-\|z\| \leq t \leq\|z\|\} \subset \mathrm{M}$ contains no pair of conjugate points of $\gamma$

b) the structure $\mathbf{P}^{\prime}$ is uniquely determined in $\gamma_{*}\left(\triangle_{\|z\|}\right)$ by the Riemannian metric $g$.

To see a) assume that $\{\gamma(a), \gamma(b)\}$ with $-\|z\| \leq a<b \leq\|z\|$ is a pair of conjugate points of $\gamma$, so, there is a non-identically zero Jacobi field $J$ along $\gamma$ which vanishes at those two points. Thus, $[\tilde{J}]_{\mathbf{P}^{\prime}}^{-}=0$ along the (image under $\gamma_{*}$ of the) segment in $\triangle_{\|z\|}$ with slope $\pi / 4$ starting at the point $(a, 0) \in \mathbb{R}^{2}$, while $[\tilde{J}]_{\mathbf{P}^{\prime}}^{+}=0$ along the (image under $\gamma_{*}$ of the) segment in $\triangle_{\|z\|}$ with slope $3 \pi / 4$ starting at the point $(b, 0) \in \mathbb{R}^{2}$. At the point of $\triangle_{\|z\|}$ where those segments meet it would follow that $\mathbf{P}^{\prime} \tilde{J}=0$, which is impossible, since the canonical extension $\tilde{J}$ along $\mathcal{L}_{\gamma}$ never vanishes off $\gamma$, and $\mathbf{P}^{\prime}$ is non-singular.

To see b), let $z_{0}=y_{0} \dot{\gamma}\left(x_{0}\right) \in \gamma_{*}\left(\triangle_{\|z\|}\right)$ with $y_{0}>0$, where, using the parametrization as before, $w=\left(x_{0}, y_{0}\right) \in \triangle_{\|z\|}$. Since $-\|z\| \leq x_{0} \leq x_{0}+y_{0} \leq\|z\|$, then $\gamma\left(x_{0}\right)$ and $\gamma\left(x_{0}+y_{0}\right)$ do not form a pair of conjugate points of $\gamma$, and thus we can find $n$ linearly independent Jacobi fields $J_{i}$ along $\gamma$ that vanish at $\gamma\left(x_{0}+y_{0}\right)$. Proposition 2.5 applied to the canonical extensions $\tilde{J}_{i}$, which are always point-wise linearly independent on $\mathcal{L}_{\gamma} \backslash \gamma$, shows that $\left[\tilde{J}_{i}(w)\right]_{\mathbf{P}^{\prime}}^{+}=0$, hence the -1-eigensubspace of $\left(T_{w}(T \mathrm{M}), \mathbf{P}^{\prime}\right)$ is spanned by $\left\{\tilde{J}_{i}(w), 1 \leq i \leq n\right\}$. Similarly, $-\|z\| \leq x_{0}-y_{0} \leq$ $x_{0} \leq\|z\|$, and by a) we can find linearly independent Jacobi fields $G_{i}$ that vanish at $\gamma\left(x_{0}-y_{0}\right)$, so that $\left[\tilde{G}_{i}(w)\right]_{\mathbf{P}^{\prime}}^{-}=0$; hence $\left\{\tilde{G}_{i}(w), 1 \leq i \leq n\right\}$ span the +-eigenspace of $\left(T_{w}(T \mathrm{M}), \mathbf{P}^{\prime}\right)$.

Remark 2.6. The result from [1 alluded to shows the equality of the structures on open serrate sets containing the zero section, which was sufficient to prove the naturality of $\mathrm{P}$ under reduction. Here, we need to show the uniqueness of $\mathbf{P}$ on any 'triangular' piece of a given Riemannian leaf of a geodesic of $\mathrm{B}$, that piece contained in an open set not necessarily serrate where $\mathbf{P}$ is defined. 


\section{Proof of Theorem 1.2}

In this section we prove Theorem 1.2 which is stated in the Introduction. It is the converse of Theorem 3 in [1]. The proof will follow from Propositions 3.4 through 3.8 ,

Proposition 3.1. Let a Lie group $G$ act on $(\mathrm{M}, g)$ by isometries freely and properly with Riemannian principal G-bundle $f_{G}: \mathrm{M} \rightarrow\left(\mathrm{B}_{G}=M / G, g_{\mathrm{B}_{G}}\right)$. Let $H \subset G$ be a Lie subgroup normal in $G$. Then we have the following $H$ and $G / H$ principal bundles and an isometric identification:

$$
\begin{array}{ccc}
(\mathrm{M}, g) & \stackrel{H}{f_{H}} & \left(\mathrm{~B}_{H}=M / H, g_{\mathrm{B}_{H}}\right) \\
G \mid f_{G} & & f_{G / H} \downarrow G / H \\
\left(\mathrm{~B}_{G}=\right. & \left.\mathrm{M} / G, g_{\mathrm{B}_{G}}\right) \stackrel{\text { isometry }}{\cong}\left(\mathrm{B}_{G / H}=\mathrm{B}_{H} / G / H, g_{\mathrm{B}_{G / H}}\right)
\end{array}
$$

Remark 3.2. Recall that a Lie subgroup $H \subset G$ of a Lie group $G$ is both a subgroup and a submanifold of $G$, and here is always assumed closed.

Corollary 3.3. Let $G$ be a Lie group acting on $(\mathrm{M}, g)$ freely and properly by isometries. Let $G_{0} \subset G$ be its connected component containing the identity. Then, with respect to the Riemannian submersion metrics, $\left(\mathrm{B}=M / G, g_{\mathrm{B}}\right)$ has no conjugate points if and only if $\left(\mathrm{B}_{0}=M / G_{0}, g_{\mathrm{B}_{0}}\right)$ has no conjugate points.

Proof. For the proposition: That $H$ acts properly and freely is standard (see [4] for instance), and the isometric identification is easy to check from the definitions. So, we leave out the details. For the corollary: It is well-known that $G_{0}$ is a normal Lie subgroup of $G$ and $G / G_{0}$ is a Lie group with $\operatorname{dim}\left(G / G_{0}\right)=0$. Putting $G_{0}=H$ in the diagram (3.1) the map $f_{G / G_{0}}$ is a Riemannian cover, in particular a local isometry. It follows that $\mathrm{M} / G$ and $\mathrm{M} / G_{0}$ are locally isometric.

Proposition 3.1 will be used in Theorem 6.1 later on. At this point, note that by Corollary 3.3 there is no loss of generality if we assumed $G$ is connected in the proof of Theorem 1.2. This is consistent with our characterization of the creation of conjugate points by quotients in terms of the moment map $\mu_{G}$, whose definition depends only on the action vector fields on $\mathrm{M}$, hence only on $G_{0}$.

3.0.1. Setup for the $G$-action on $\mu_{G}{ }^{-1}(0)$ in relation to $\mathbf{P}$.

For $\xi$ in $\mathfrak{G}$, the Lie Algebra of $G$, we denote by $\xi_{\mathrm{M}}$ the action vector field on $\mathrm{M}$ and by $\xi_{T \mathrm{M}}$ the action vector field on $T \mathrm{M}$ corresponding to the tangential $G$ action. With this notation we express the moment map of the tangential action $\mu_{G}: T \mathrm{M} \rightarrow \mathfrak{G}^{*}$ evaluated at $z \in T \mathrm{M}$ and applied to $\xi \in \mathfrak{G}$ as

$$
\mu_{G}(z)[\xi]=\Theta\left(\xi_{T \mathrm{M}}(z)\right)=g\left(\xi_{\mathrm{M}}(\pi(z))\right) .
$$

Now, $(\mathbf{M}, g)$ have no conjugate points by hypothesis, so $\mathbf{P}$ is defined in the entire $T \mathrm{M}$. Let $\mathfrak{G}^{T \mathrm{M}}$ and $\mathbf{P} \mathfrak{G}^{T \mathrm{M}}$ be the distributions on $T \mathrm{M}$ defined by the tangential action on $T \mathrm{M}$ and $\mathbf{P}$,

$$
\left.\mathfrak{G}^{T \mathrm{M}}\right|_{z}=\left\{\zeta_{T \mathrm{M}}(z) \mid \zeta \in \mathfrak{G}\right\},\left.\quad \mathbf{P G}^{T \mathrm{M}}\right|_{z}=\left\{\mathbf{P} \zeta_{T \mathrm{M}}(z) \mid \zeta \in \mathfrak{G}\right\} .
$$

These two distributions each have rank that equals $\operatorname{dim} G$ since the action on $\mathrm{M}$ is free. It follows that the tangential action on $T \mathrm{M}$ is free as well. 
Proposition 3.4. For all $z \in T \mathrm{M}$

$$
\left.\mathfrak{G}^{T \mathrm{M}}\right|_{z} \cap\left(\left.\mathbf{P} \mathfrak{G}^{T \mathrm{M}}\right|_{z}\right)=\{0\} .
$$

Proof. We sketch the proof and refer to [1] for more details. Use (2.8) for $z$ on the zero section. On the other hand, if $z \neq 0$ use the fact that along any geodesic $\gamma$ of $\mathrm{M}$ any action vector field $\zeta_{\mathrm{M}}$ for the $G$-action on M restricts to a Jacobi vector field $\zeta_{\gamma}$ along $\gamma$. Then, Proposition 2.5 and the hypothesis that the $G$-action on M is free imply that the canonical extension $\tilde{\zeta}_{\gamma}$ of $\zeta_{\gamma}$ along $\mathcal{L}_{\gamma}$ is at no point of $\mathcal{L}_{\gamma}$ an eigenvector of $\mathbf{P}$. The linearity of the Jacobi equation gives (3.4).

We define the distribution $H$ on $T \mathrm{M}$ as

$$
H=\left(\mathfrak{G}^{T \mathrm{M}}+\mathbf{P} \mathfrak{G}^{T \mathrm{M}}\right)^{\perp_{d \Theta}},
$$

where $\perp_{d \Theta}$ indicates orthogonal subspace with respect to the symplectic form $d \Theta$. This distribution is $G$-invariant since $d \Theta$ and $\mathbf{P}$ are $G$-invariant. It is also $\mathbf{P}$ invariant, since $d \Theta=d \mathbf{P} d E$ is anti-P-invariant, i.e., $d \Theta(\mathbf{P} U, \mathbf{P} U)=-d \Theta(U, U)$, as its expression in local product coordinates shows. (See [1] for details.)

Proposition 3.5. The $G$-action is tangentially positive at $z \in \mu_{G}{ }^{-1}(0)$ if and only if

$$
T_{z}\left(\mu_{G}{ }^{-1}(0)\right)=\mathfrak{G}_{z}^{T \mathrm{M}} \oplus H_{z} .
$$

Proof. We sketch the proof and again refer to [1] for additional details. From Proposition 3.4 and the fact that the symplectic form is non-degenerate it follows that $\operatorname{dim} H+\operatorname{dim} H^{\perp_{d \Theta}}=2 n$ at any $z \in T \mathrm{M}$. Consequently the $G$-action is tangentially positive at $z \in T \mathrm{M}$ if and only if $T_{z}(T \mathrm{M})=H_{z}^{\perp} d \Theta \oplus H_{z}=\left(\mathfrak{G}_{z}^{T \mathrm{M}}+\mathbf{P} \mathfrak{G}_{z}^{T \mathrm{M}}\right) \oplus H_{z}$.

But, if now $z \in \mu_{G}{ }^{-1}(0)$, we have $T_{z}\left(\mu_{G}{ }^{-1}(0)\right)=\left(\mathfrak{G}_{z}^{T \mathrm{M}}\right)^{\perp_{d} \Theta} \supset \mathfrak{G}_{z}^{T \mathrm{M}}$, and thus, at such $z$, the tangential positivity condition for the action reads $\mathbf{P} \mathfrak{G}_{z}^{T \mathrm{M}} \cap$ $T_{z}\left(\mu_{G}{ }^{-1}(0)\right)=\{0\}$.

Now, let $\left\{\zeta^{1}, \ldots, \zeta^{\operatorname{dim} G}\right\}$ be a basis for $\mathfrak{G}$ and consider the matrix-valued function on $T \mathrm{M}$ with entries $d \Theta\left(\mathbf{P} \zeta_{T \mathrm{M}}^{i}(z), \zeta_{\mathrm{M}}^{j}(z)\right)$. Put

$$
\mathcal{C} \stackrel{\text { def }}{=}\left\{z \in T \mathrm{M} \mid \operatorname{rank}\left(d \Theta\left(\mathbf{P} \zeta_{T \mathrm{M}}^{i}(z), \zeta_{\mathrm{M}}^{j}(z)\right)\right)=0\right\} .
$$

Proposition 3.6. If the $G$-action is free, $\mathcal{C} \cap \mathrm{M}=\emptyset$.

Proof. At each $0=z=0_{P} \in T_{P} \mathrm{M}$, we have, from (2.11) and under the identification of $\mathrm{M}$ with the zero section of $T \mathrm{M}$, that $\zeta_{T \mathrm{M}}\left(0_{p}\right)=\left(\zeta_{\mathrm{M}}(p)\right)_{0_{p}}^{h}$. Thus, by (2.4) and (2.8),

$$
\left.d \Theta\left(\mathbf{P} \zeta_{T \mathrm{M}}^{i}(z), \zeta_{T \mathrm{M}}^{j}(z)\right)\right|_{z=0_{p}}=d \Theta\left(\mathbf{P}\left(\zeta_{\mathrm{M}}^{i}(p)\right)_{0_{p}}^{v},\left(\zeta_{\mathrm{M}}^{j}(p)\right)_{0_{p}}^{h}\right)=g\left(\zeta_{\mathrm{M}}^{i}(p), \zeta_{\mathrm{M}}^{j}(p)\right) .
$$

But, since the action on $\mathrm{M}$ is free, the $\operatorname{dim} G \times \operatorname{dim} G$ matrix-valued function on M with entries $g\left(\zeta_{\mathrm{M}}^{i}, \zeta_{\mathrm{M}}^{j}\right)$ is positive-definite.

Now, put

$$
\mathcal{O}^{\dagger} \stackrel{\text { def }}{=} T \mathrm{M} \backslash \mathcal{C}
$$


which is open in $T \mathrm{M}$ and contains the zero section by Proposition 3.6. $G$ acts on the (in general, disconnected) open set $\mathcal{O}^{\dagger} \subset T \mathrm{M}$ and on the closed set $\mu_{G}{ }^{-1}(0) \subset T \mathrm{M}$, and thus, the distribution $\left.H\right|_{\mathcal{O}}{ }^{+}$serves as the horizontal subspace of a connection for the principal $G$-bundle

$$
\mathcal{O}^{\dagger} \cap \mu_{G}^{-1}(0) \rightarrow\left(\mathcal{O}^{\dagger} \cap \mu_{G}{ }^{-1}(0)\right) / G .
$$

By the $G$ and $\mathbf{P}$-invariance of $H$ we can 'push-down' $\mathbf{P}$ and define an integrable product structure $\check{\mathbf{P}}$ on $\left(\mathcal{O}^{\dagger} \cap \mu_{G}{ }^{-1}(0)\right) / G \subset T$ B. The inclusion is understood via the identification $\mu_{G}{ }^{-1}(0) / G=T \mathrm{~B}$, with $\left(\mathcal{O}^{\dagger} \cap \mu_{G}{ }^{-1}(0)\right) / G$ open in $T \mathrm{~B}$ (since $\mu_{G}{ }^{-1}(0)$ is closed in $\left.T \mathrm{M}\right)$ and containing B. Integrability of $\check{\mathbf{P}}$ is expressed in the identity $[\check{\mathbf{P}} U, \check{\mathbf{P}} V]+[U, V]=\check{\mathbf{P}}[U, \check{\mathbf{P}} V]+\check{\mathbf{P}}[\check{\mathbf{P}} U, V]$. To show it, due to the integrability of $P$ it suffices to prove that if $X$ and $Y$ are $G$-invariant local sections of $H$ in $\mu_{G}{ }^{-1}(0) \cap \mathcal{O}^{\dagger}$, then $W=[X, Y]+[\mathbf{P} X, \mathbf{P} Y]$ is also a $G$-invariant section of $H$. (Proof: Since $\mathbf{P}$ is $G$-invariant, so is $W$. Also, from $d^{2} \Theta=0$ we have, for all $\xi \in \mathfrak{G}, 0=d^{2} \Theta\left(X, Y, \xi_{T \mathrm{M}}\right)+d^{2} \Theta\left(\mathbf{P} X, \mathbf{P} Y, \xi_{T \mathrm{M}}\right)$. Use this, as well as $d \Theta(X, Y)+d \Theta(\mathbf{P} X, \mathbf{P} Y)=0$, that the Lie derivative $\mathbf{L}_{\xi_{T M}} d \Theta=0$, and that $d \Theta\left(X, \xi_{T \mathrm{M}}\right)=d \Theta\left(Y, \xi_{T \mathrm{M}}\right)=0$. Also, see [1].)

Let $\mathcal{O}_{0}^{\dagger}$ be the connected component of $\mathcal{O}^{\dagger}$ containing the zero section of $T \mathrm{M}$. Clearly $\mathcal{O}_{0}^{\dagger}$ is also invariant by the tangential $G$-action, since $h_{*}$ is a homeomorphism of $T \mathrm{M}$ if $h \in G$ is a homeomorphism of M. Hence $\left(\mathcal{O}_{0}^{\dagger} \cap \mu_{G}{ }^{-1}(0)\right) / G \subset T \mathrm{~B}$ is an open connected set containing $\mathrm{B}$ where both $\check{\mathbf{P}}$ and $\mathbf{P}_{\mathrm{B}}$ are defined.

Now, let $i_{\mu_{G}}: \mu_{G}^{-1}(0) \rightarrow T \mathrm{M}$ be the inclusion and put

$$
\mathfrak{p}=f_{*} \circ i_{\mu_{G}}: \mu_{G}{ }^{-1}(0) \rightarrow T \mathrm{~B} .
$$

By the classical symplectic reduction process ([14]) we 'push-down' the one-form $\Theta$ and the energy function $E$ on $T \mathrm{M}$ to a one-form $\Theta$ and a function $\check{E}$ on $T \mathrm{~B}$, so that

$$
\mathfrak{p}^{*} \check{\Theta}=\left(i_{\mu_{G}}\right)^{*} \Theta, \quad \mathfrak{p}^{*} E=\left(i_{\mu_{G}}\right)^{*} E, \text { on } \mu_{G}{ }^{-1}(0) \text {. }
$$

Moreover, it follows that $\check{\Theta}=\Theta_{\mathrm{B}}$ and $\check{E}=E_{\mathrm{B}}$ on $T$ B.

For $z \in \mathcal{O}^{\dagger} \cap \mu_{G}{ }^{-1}(0)$, where $H_{z}$ defines the horizontal subspace of a connection, we have that

$$
\mathfrak{p}_{*}:\left(H_{z},\left.d \Theta\right|_{H z}\right) \rightarrow\left(T_{\mathfrak{p} z} \mathrm{~B}, d \Theta_{\mathrm{B}}\right)
$$

is a symplectic isomorphism. In particular, since the geodesic spray $\Sigma$ and the Liouville vector field $\Xi$ satisfy $\mathbf{P} \Sigma(z)=\Xi(z)$ for all $z \in T \mathrm{M}$, while $\Sigma(z) \in H_{z}$ and $\Xi(z) \in H_{z}$ for all $z \in \mu_{G}{ }^{-1}(0)$, the pushed down equation $\check{\mathbf{P}} d \check{E}=\check{\Theta}$ holds on $\left(\mathcal{O}^{\dagger} \cap \mu_{G}^{-1}(0)\right) / G$, in particular on $\left(\mathcal{O}_{0}^{\dagger} \cap \mu_{G}{ }^{-1}(0)\right) / G$.

Thus, $\check{\mathbf{P}}^{2}=I, \check{\mathbf{P}}$ is integrable, and $\check{\mathbf{P}} d E_{\mathrm{B}}=\Theta_{\mathrm{B}}$ on $\left(\mathcal{O}_{0}^{\dagger} \cap \mu_{G}{ }^{-1}(0)\right) / G$. We have the following.

Proposition 3.7. Let $\mathcal{S} \subset \mathcal{O}_{0}^{\dagger} \subset T \mathrm{M}$ be a serrate set. Then

$$
\check{\mathbf{P}}=\mathbf{P}_{\mathrm{B}} \text { on } \mathfrak{p}\left(\mathcal{S} \cap \mu_{G}{ }^{-1}(0)\right),
$$

that is, they agree as endomorphisms of $T_{w}(T \mathrm{~B})$ for all $w \in \mathfrak{p}\left(\mathcal{S} \cap \mu_{G}{ }^{-1}(0)\right)$.

Proof. It suffices to show that for any serrate set $\mathcal{S} \subset T \mathrm{M}$

$$
f_{*}\left(\mathcal{S} \cap \mu_{G}{ }^{-1}(0)\right)
$$


is a serrate set of $T \mathrm{~B}$ and then apply Proposition 2.3 to $\left(\mathrm{B}, g_{\mathrm{B}}\right)$, because the set $\left(\mathcal{O}_{0}^{\dagger} \cap \mu_{G}{ }^{-1}(0)\right) / G$, where both $\check{\mathbf{P}}$ and $\mathbf{P}_{\mathrm{B}}$ are defined, contains $\mathfrak{p}\left(\mathcal{S} \cap \mu_{G}{ }^{-1}(0)\right)$, is open in $T \mathrm{~B}$ and contains $\mathrm{B}$.

According to Definition 2.1 and 2.2 applied to B we need to show that given $w=f_{*} z \in T \mathrm{~B}$ with $0 \neq z \in \mathcal{S} \cap \mu_{G}{ }^{-1}(0)$ we have

$$
\left(\delta_{w}\right)_{*}\left(\triangle_{\|w\|_{\mathrm{B}}}\right) \subset \mathfrak{p}\left(\mathcal{S} \cap \mu_{G}^{-1}(0)\right),
$$

where $\|\cdot\|_{\mathrm{B}}$ is the length in B. Here we assume $z \neq 0$ because $\mathfrak{p}\left(\mathcal{S} \cap \mu_{G}{ }^{-1}(0) \cap \mathrm{M}\right) \subset$ $\mathrm{B}$, and clearly any subset of the zero section $\mathrm{B}$ is serrate. Also, $w=f_{*} z \neq 0$ since $z \in \mu_{G}{ }^{-1}(0) \backslash \mathrm{M}$. Thus, in what follows we let $\delta_{w}: \mathbb{R} \rightarrow \mathrm{B}$ be the unit-speed geodesic such that $\|w\|_{\mathrm{B}} \dot{\delta}_{w}(0)=w$ and let $\gamma_{z}: \mathbb{R} \rightarrow \mathrm{M}$ be the unit-speed geodesic with $\|z\|_{\mathrm{M}} \dot{\gamma}_{z}(0)=z$.

Since $\mu_{G}{ }^{-1}(0)$ is a vector subbundle of the $T \mathrm{M}$ invariant by the geodesic flow, it is a serrate set; in fact

$$
\mu_{G}{ }^{-1}(0)=\bigcup_{f \text {-horizontal geodesic } \gamma} \mathcal{L}_{\gamma},
$$

where, we recall, a geodesic if $f$-horizontal if it is point-wise orthogonal to the action vector fields of the $G$-action in M, that is, to the fibers of the principal bundle $f: \mathrm{M} \rightarrow \mathrm{B}$. But a geodesic that we know is orthogonal to the fibres at one point must be $f$-horizontal (since, as a consequence of the skew-symmetry of $\nabla \xi_{\mathrm{M}}$ for any action vector field, $g\left(\xi_{\mathrm{M}}(\gamma(t)), \dot{\gamma}(t)\right)$ is independent of $t$ for any constantspeed geodesic $\gamma$ ). Thus, since $\|z\|_{\mathrm{M}} \dot{\gamma}_{z}(0)=z \in \mu_{G}^{-1}(0)$, it follows that $\gamma_{z}$ is $f$-horizontal.

So, since the intersection of serrate sets is a serrate set, $\mathcal{S} \cap \mu_{G}{ }^{-1}(0)$ is a serrate set and thus, by the hypothesis on $\mathcal{S}$ and the definition of serrate set,

$$
\left(\gamma_{z}\right)_{*}\left(\triangle_{\|z\|_{\mathrm{M}}}\right) \subset \mathcal{S} \cap \mu_{G}{ }^{-1}(0)
$$

But since $\gamma_{z}$ is $f$-horizontal we have that $f \circ \gamma_{z}: \mathbb{R} \rightarrow \mathrm{B}$ is a geodesic of $\mathrm{B}$ such that $\left(f \circ \gamma_{z}\right)(0)=f_{*} z=w$, and hence $f \circ \gamma_{z}=\delta$, by the uniqueness of a geodesic with given initial conditions. Moreover, $\|w\|_{\mathrm{B}}=\left\|f_{*} z\right\|_{\mathrm{B}}=\|z\|_{\mathrm{M}}$ and so,

$$
f_{*}\left(\gamma_{z}\right)_{*}\left(\triangle_{\|z\|_{\mathrm{M}}}\right)=\left(f \circ \gamma_{z}\right)_{*}\left(\triangle_{\|z\|_{\mathrm{M}}}\right)=\left(\delta_{w}\right)_{*}\left(\triangle_{\|w\|_{\mathrm{B}}}\right)
$$

which applied to (3.14) shows (3.12) as required.

Proposition 3.8. Let $\mathcal{K} \subset T \mathrm{M} \backslash \mathrm{M}$ be a closed set. Let $\gamma$ be a unit-speed geodesic whose Riemannian leaf $\mathcal{L}_{\gamma} \cap \mathcal{K} \neq \emptyset$. Consider the parametrization $\gamma_{*}: \mathbb{R}^{2} \rightarrow \mathcal{L}_{\gamma}$, $\gamma_{*}(x, y) \mapsto y \dot{\gamma}(x)$. Then, using notation (2.3), after possibly a change of origin of the parameter of $\gamma$, there is an $0<r \in \mathbb{R}$ such that

$$
\gamma_{*}\left(\triangle_{t}\right) \cap \mathcal{K}=\left\{\begin{array}{l}
\emptyset, \text { if } 0 \leq t<r, \\
\left\{\gamma_{*}(0, r)\right\}, \text { if } t=r .
\end{array}\right.
$$

Proof. It is enough to show that for the given parametrization of $\gamma$ there are $a<b \in$ $\mathbb{R}$ such that $\triangle$, the convex hull of $\left\{(a, 0),(b, 0),\left(\frac{a+b}{2}, \frac{b-a}{2}\right)\right\} \subset \mathrm{R}^{2}$, satisfies $\gamma_{*}(\triangle) \cap$ $\mathcal{K}=\gamma_{*}\left(\frac{a+b}{2}, \frac{b-a}{2}\right)=\left(\frac{b-a}{2}\right) \gamma\left(\frac{a+b}{2}\right)$, for then we perform an obvious translation in the arc-length parameter of $\gamma$ if necessary. To start pick $z \in \mathcal{L}_{\gamma} \cap \mathcal{K}$ and set the arc-length parameter of $\gamma$ so that $\gamma(0)=\pi(z)$ to define

$$
T=\inf \left\{t \in \mathbb{R} \mid \gamma_{*}\left(\triangle_{t}\right) \cap \mathcal{K} \neq \emptyset\right\} .
$$


Since $\mathcal{K}$ is closed and is disjoint from the zero section $\mathrm{M}$, it holds that $T>0$,

$$
\emptyset \neq \triangle_{T} \cap \gamma_{*}^{-1} \mathcal{K} \subset \text { boundary of } \triangle_{T},
$$

and $0<h \leq T$ where

$$
h \stackrel{\text { def. }}{=} \inf _{\left\{z \in \gamma_{*}\left(\triangle_{T}\right) \cap \mathcal{K}\right\}}\|z\| .
$$

By (3.17) and (3.18) it follows that

$$
\emptyset \neq \mathcal{K} \cap \gamma_{*}\left(\triangle_{T}\right) \cap\{\|z\| \leq h\} \subset\left\{\gamma_{*}(h-T, h), \gamma_{*}(T-h, h)\right\} \subset \mathcal{L}_{\gamma} .
$$

Thus, our $\triangle$ as stated at the beginning of the proof will be the convex hull of $\{(-T, 0),(2 h-T, 0),(h-T, h)\} \subset \mathbb{R}^{2}$ or of $\{(T-2 h, 0),(T, 0),(T-h, h)\} \subset \mathbb{R}^{2}$.

\subsection{Proof of Theorem 1.2.}

Proof. We assume that the $G$-action on $(\mathrm{M}, g)$ is not tangentially positive in all of $\mu_{G}{ }^{-1}(0)$ and that $\left(\mathrm{B}, g_{\mathrm{B}}\right)$ has no conjugate points, hence $P_{\mathrm{B}}$ is defined in all $T \mathrm{~B}$, and we derive from this a contradiction.

Thus, by hypothesis $\mathcal{C} \cap \mu_{G}{ }^{-1}(0) \neq \emptyset$, where the set $\mathcal{C} \subset T \mathrm{M}$ is given by (3.7), and clearly independent of the choice of basis for $\mathfrak{G}$. Accordingly, we have the following.

Proposition 3.9. Given $z \in \mathcal{C} \cap \mu_{G}{ }^{-1}(0)$ there is a $\psi \in \mathfrak{G}$ such that

$$
\psi_{T \mathrm{M}}(z) \in H_{z}=\left(\mathfrak{G}_{z}^{T \mathrm{M}}+\mathbf{P} \mathfrak{G}_{z}^{T \mathrm{M}}\right)^{\perp_{d \Theta}} .
$$

Thus, putting $U=\mathbf{P} \psi_{T \mathrm{M}}(z) \in T_{z}(T \mathrm{M})$ we have:

1) $U \in H_{z}$,

2) $\mathfrak{p}_{*} U \neq 0$,

3) $\mathfrak{p}_{*} \mathbf{P} U=0$.

Proof. First note that 1) follows from (3.19) since $H$ is $\mathbf{P}$-invariant. Also, 2) and 3) follow the facts that $U \in \mathbf{P} \mathfrak{G}_{z}^{T \mathrm{M}}, \mathbf{P} U \in \mathfrak{G}_{z}^{T \mathrm{M}}$, ker $\mathfrak{p}_{*}=\mathfrak{G}^{T \mathrm{M}}$, and $\mathfrak{G}^{T \mathrm{M}} \cap \mathbf{P} \mathfrak{G}^{T \mathrm{M}}=$ $\{0\}$ by (3.4).

We now show (3.19). Since $z \in \mathcal{C}$ there is $\xi$ and $\psi \in \mathfrak{G}$, such that

$$
0 \neq \xi_{T \mathrm{M}}(z)+\mathbf{P} \psi_{T \mathrm{M}}(z) \in\left(\mathfrak{G}_{z}^{T \mathrm{M}}+\mathbf{P G}_{z}^{T \mathrm{M}}\right) \cap\left(\mathfrak{G}_{z}^{T \mathrm{M}}+\mathbf{P G}_{z}^{T \mathrm{M}}\right)^{\perp_{d \ominus}} .
$$

Since $d \Theta$ is anti-P-invariant, $H^{\perp_{d \Theta}} \cap H$ is $\mathbf{P}$-invariant. Thus we assume without loss of generality that in (3.20) $\psi_{P \mathrm{M}}(z) \neq 0$ since otherwise we consider the image by $\mathbf{P}$ of the vector in (3.20). But, recall that $d \Theta$ restricts to the zero two-form on $\mathfrak{G}_{z}^{T \mathrm{M}}$ if $z \in \mu_{G}{ }^{-1}(0)$, since $T_{z}\left(\mu_{G}^{-1}(0)\right)=\left(\mathfrak{G}_{z}^{T \mathrm{M}}\right)^{\perp_{d \Theta}}$ (i.e., $\mathfrak{G}_{z}^{T \mathrm{M}}$ is "isotropic" with respect to $d \Theta$ at such $z$ ), thus,

$$
0=d \Theta\left(\psi_{T \mathrm{M}}(z), \zeta_{T \mathrm{M}}(z)\right), \quad \forall \zeta \in \mathfrak{G} .
$$

Also, using (3.20), and again, in Theorem $\left({ }^{*}\right)$, the 'isotropy' of $\mathfrak{G}_{z}^{T \mathrm{M}}$ with respect to $d \Theta$,

$$
\begin{aligned}
0 & =d \Theta\left(\xi_{T \mathrm{M}}(z)+\mathbf{P} \psi_{T \mathrm{M}}(z), \zeta_{T \mathrm{M}}(z)\right) \\
& \stackrel{(*)}{=} d \Theta\left(\mathbf{P} \psi_{T \mathrm{M}}(z), \zeta_{T \mathrm{M}}(z)\right) \\
& =-d \Theta\left(\psi_{T \mathrm{M}}(z), \mathbf{P} \zeta_{T \mathrm{M}}(z)\right), \quad \forall \zeta \in \mathfrak{G} .
\end{aligned}
$$

So, by (3.21), (3.22) and (3.5), $\psi_{T \mathrm{M}}(z)$ is in $H_{z}$, and the claim is proved. 
Since $\mathcal{C}$ and $\mu_{G}{ }^{-1}(0)$ are both closed in $T \mathrm{M}$, their intersection is also closed. Moreover, since by hypothesis the $G$-action is free, $\mathcal{C} \cap \mathrm{M}=\emptyset$ from Proposition 3.6. and $\mathcal{C} \cap \mu_{G}{ }^{-1}(0) \cap M=\emptyset$. Thus, recalling (3.13), it follows that there is a unit-speed $f$-horizontal geodesic $\gamma_{z^{c}}: \mathbb{R} \rightarrow \mathrm{M}$ such that $\mathcal{L}_{\gamma_{z^{c}}} \cap \mathcal{C} \cap \mu_{G}{ }^{-1}(0) \neq \emptyset$. Consequently, we may apply Proposition 3.8 to

$$
\mathcal{K} \stackrel{\text { def }}{=} \mathcal{C} \cap \mu_{G}{ }^{-1}(0) \neq \emptyset .
$$

We conclude that there is $0<r \in \mathbb{R}$, and $\triangle_{r} \subset \mathbb{R}^{2}$ as above, with choice of origin for the arc-length parameter of $\gamma_{z^{c}}$ such that, putting

$$
z^{c}=\left(\gamma_{z^{c}}\right)_{*}(0, r)=r \dot{\gamma}_{z^{c}}(0)
$$

with $r=\left\|z^{c}\right\|$, we have

1) $\mathcal{L}_{\gamma_{z^{c}}} \subset \mu_{G}{ }^{-1}(0) \quad$ (by (3.13), since $\gamma_{z^{c}}$ is $f$-horizontal);

2) $\left(\gamma_{z^{c}}\right)_{*}\left(\triangle_{r}\right) \cap \mathcal{C}=\left\{z^{c}\right\}$;

3) $\left(\gamma_{z^{c}}\right)_{*}\left(\triangle_{s}\right) \subset \mathcal{O}_{0}^{\dagger}, \forall s \mid 0 \leq s<r$.

Hence, since $\left(\gamma_{z^{c}}\right)_{*}\left(\triangle_{s}\right)$ is serrate, and by 1$)$ we have $\left(\gamma_{z^{c}}\right)_{*}\left(\triangle_{s}\right) \subset \mu_{G}^{-1}(0)$ for any $s \geq 0$ while by 3$)$ it holds that $\left(\gamma_{z^{c}}\right)_{*}\left(\triangle_{s}\right) \subset \mathcal{O}_{0}^{\dagger}$ for all $0 \leq s<r$, it follows by Proposition 3.7 that

$$
\mathfrak{p}_{*}:\left(H_{z}, \mathbf{P}\right) \rightarrow\left(T_{\mathfrak{p} z}(T \mathrm{~B}), \mathbf{P}_{\mathrm{B}}\right)
$$

is a product structure preserving linear isomorphism for all $z \in\left(\gamma_{z^{c}}\right)_{*}\left(\triangle_{s}\right)$ for all $0 \leq s<r$.

In particular for all $t \in[0,1)$,

$$
\mathfrak{p}_{*}:\left(H_{t z^{c}}, \mathbf{P}\right) \rightarrow\left(T_{t w^{c}}(T \mathrm{~B}), \mathbf{P}_{\mathrm{B}}\right)
$$

is product structure preserving, where we put $w^{c}=\mathfrak{p} z^{c}=f_{*} z^{c}$.

Now let $X:[0,1] \rightarrow H \subset T(T \mathrm{M})$ be continuous such that

$$
X(t) \in H_{t z^{c}}, \forall t \in[0,1] \text { with } X(1)=U .
$$

Given any continuous $Y:[0,1] \rightarrow T\left(\mu_{G}{ }^{-1}(0)\right)$ such that

$$
Y(t) \in T_{t z^{c}}\left(\mu_{G}^{-1}(0)\right), \quad \forall t \in[0,1],
$$

define the continuous functions $f_{Y}:[0,1] \rightarrow \mathbb{R}$ and $k_{Y}:[0,1] \rightarrow \mathbb{R}$ by

$$
\begin{aligned}
& f_{Y}(t) \stackrel{\text { def }}{=} d \Theta(\mathbf{P} X(t), Y(t)), \\
& k_{Y}(t) \stackrel{\text { def }}{=} d \Theta_{\mathrm{B}}\left(\mathbf{P}_{\mathrm{B}} \mathfrak{p}_{*} X(t), \mathfrak{p}_{*} Y(t)\right) .
\end{aligned}
$$

But, $H$ is $\mathbf{P}$-invariant, so

$$
\mathbf{P} X(t) \in H_{t z^{c}} \subset T_{t z^{c}}\left(\mu_{G}{ }^{-1}(0)\right), \quad \forall t \in[0,1],
$$

where the inclusion $H_{t z^{c}} \subset T_{t z^{c}}\left(\mu_{G}{ }^{-1}(0)\right)$ holds since $t z^{c} \in \mu_{G}^{-1}(0)$ for all $t$. Thus, since also $Y(t) \in H_{t z^{c}}$ for all $t \in[0,1]$ and $i_{\mu_{G}}^{*} d \Theta=\mathfrak{p}^{*} d \Theta_{\mathrm{B}}$, we have the equality

$$
f_{Y}(t)=d \Theta_{\mathrm{B}}\left(\mathfrak{p}_{*} \mathbf{P} X(t), \mathfrak{p}_{*} Y(t)\right), \quad \forall t \in[0,1] .
$$

In particular, from part 3) of Proposition 3.9 and (3.25), for any section $Y$ as in (3.26),

$$
f_{Y}(1)=d \Theta_{\mathrm{B}}\left(\mathfrak{p}_{*} \mathbf{P} U, \mathfrak{p}_{*} Y(1)\right)=0 .
$$


But, by (3.24)

$$
\mathfrak{p}_{*} \circ \mathbf{P}=\mathbf{P}_{\mathrm{B}} \circ \mathfrak{p}_{*}: H_{t z^{c}} \rightarrow T_{t w^{c}}(T \mathrm{~B}), \quad \forall t \in[0,1),
$$

and thus

$$
\begin{aligned}
f_{Y}(t) & =d \Theta(\mathbf{P} X(t), Y(t)) \\
& =d \Theta_{\mathrm{B}}\left(\mathbf{P}_{\mathrm{B}} \mathfrak{p}_{*} X(t), \mathfrak{p}_{*} Y(t)\right) \\
& =k_{Y}(t), \quad \forall t \in[0,1) .
\end{aligned}
$$

It follows by the continuity of $f_{Y}$ and of $k_{Y}$ on $[0,1]$ that

$$
0=f_{Y}(1)=k_{Y}(1)=d \Theta_{\mathrm{B}}\left(\mathbf{P}_{\mathrm{B}} \mathfrak{p}_{*} U, \mathfrak{p}_{*} Y(1)\right),
$$

for all $Y$ as in (3.26). Since $\mathfrak{p}_{*} T_{z^{c}}\left(\mu_{G}^{-1}(0)\right)=T_{w^{c}} \mathrm{~B}$ and $d \Theta_{\mathrm{B}}$ in non-degenerate, we have $\mathbf{P}_{\mathrm{B}} \mathfrak{p}_{*} U=0$. Since by part 2 ) of the claim $\mathfrak{p}_{*} U \neq 0$, we must have ker $\mathbf{P}_{\mathrm{B}} \neq\{0\}$ which is a contradiction since $\left(\mathrm{B}, g_{\mathrm{B}}\right)$ has no conjugate points. (More precisely, the above would imply that $\delta\left(-\left\|w^{c}\right\|\right)$ and $\delta\left(\left\|w^{c}\right\|\right)$ are conjugate points of the geodesic $\delta: \mathbb{R} \rightarrow \mathrm{B}$.) So we must have $\mathcal{C}=\emptyset$, and the $G$-action on $(\mathrm{M}, g)$ is tangentially positive on $\mu_{G}{ }^{-1}(0)$.

This completes the proof of Theorem 1.2 .

\subsubsection{Some remarks.}

Let us denote by $\check{G}_{\mathbf{P}}$ the pseudo-Riemannian metric on $\left(\mathcal{O}^{\dagger} \cap \mu_{G}{ }^{-1}(0)\right) / G$ defined by $d \check{\Theta}$ and $\check{\mathbf{P}}$. The above shows that for all $z \in \mathcal{O}^{\dagger} \cap \mu_{G}{ }^{-1}(0)$, the map $\mathfrak{p}_{*}:\left(H_{z},\left.\mathbf{G}_{\mathbf{P}}\right|_{H_{z}}\right) \rightarrow\left(T_{\mathfrak{p} z} \mathrm{~B}, \check{G}_{\mathbf{P}}\right)$ is an isometry, and $\mathfrak{p}:\left(\mathcal{O}^{\dagger} \cap \mu_{G}{ }^{-1}(0), i_{\mu_{G}}^{*} \mathbf{G}_{\mathbf{P}}\right) \rightarrow$ $\left(T \mathrm{~B}, \check{G}_{\mathbf{P}}\right)$ a pseudo-Riemannian submersion. Thus, in light of Proposition 3.7 we have proved

Proposition 3.10. When we give TB the metric $G_{\mathbf{P}_{\mathrm{B}}}$, and $\mathcal{O}^{\dagger} \cap \mu_{G}{ }^{-1}(0)$ the metric $i_{\mu_{G}}^{*} \mathbf{G}_{\mathbf{P}}$, then for any serrate set $\mathcal{S} \subset \mathcal{O}^{\dagger} \subset T \mathrm{M}$ the submersion

$$
\mathfrak{p}:\left(\mu_{G}^{-1}(0)\right) \rightarrow(T \mathrm{~B})
$$

is a pseudo-Riemannian submersion at every point $z \in \mathcal{S} \cap \mu_{G}{ }^{-1}(0)$.

Remark 3.11. As a particular case, if $(\mathrm{M}, g)$ has no conjugate points and the $G$ action on $\mathrm{M}$ is tangentially positive, then $\mathcal{O}^{\dagger}=T \mathrm{M}$, and we may take $\mathcal{S}=\mu_{G}{ }^{-1}(0)$ in the proposition above. Hence, in this situation,

$$
\mathfrak{p}:\left(\mu_{G}{ }^{-1}(0), i_{\mu_{G}}^{*} \mathbf{G}_{\mathbf{P}}\right) \rightarrow\left(T \mathrm{~B}, G_{\mathbf{P}_{\mathrm{B}}}\right)
$$

is a pseudo-Riemannian submersion. Incidentally note that $\mathbf{G}_{\mathbf{P}}$ has signature $(\operatorname{dim} \mathrm{B}, \operatorname{dim} \mathrm{M})$ and $G_{\mathbf{P}_{\mathrm{B}}}$ signature $(\operatorname{dim} \mathrm{B}, \operatorname{dim} \mathrm{B})$. This was remarked in [1] as a case of 'pseudo-Riemannian submersion commuting with reduction'.

\section{4. $\mathbf{G}_{\mathbf{P}}$-Length of tangential lifts of vector fields. Theorem 4.1}

In this section $(\mathrm{M}, g)$ is complete and has no conjugate points, so that $\mathbf{P}$ is defined on the whole $T \mathrm{M}$. For any vector field $X$ on $\mathrm{M}$ we define

$$
\mathcal{S}_{X} \stackrel{\text { def }}{=}\{\mathbb{R} X(p) \mid p \in \mathrm{M}\} \subset T \mathrm{M} .
$$

Let us now state Theorem 4.1 
Theorem 4.1. Let $X$ be a vector field on $\mathrm{M}$ and $X_{T \mathrm{M}}$ the vector field on $T \mathrm{M}$ induced by the tangent maps of the flow of $X$. Then

$$
\nabla_{X} X=0 \text { on } \mathrm{M} \Longleftrightarrow \mathbf{G}_{\mathbf{P}}\left(X_{T \mathrm{M}}, X_{T \mathrm{M}}\right) \geq 0 \text { on } \mathcal{S}_{X} \subset T \mathrm{M} \text {. }
$$

The proof of Theorem 4.1 follows from the next 3 propositions.

Proposition 4.2. Let $X: \mathrm{M} \rightarrow T \mathrm{M}$ and $X_{T \mathrm{M}}: T \mathrm{M} \rightarrow T(T \mathrm{M})$ be vector fields as in the statement of Theorem 4.1. Then for $z \in T_{p} \mathrm{M}$,

$$
X_{T \mathrm{M}}(z)=(X(p))_{z}^{h}+\left(\nabla_{z} X\right)_{z}^{v} .
$$

Proof. Let $t \mapsto f_{t}: \mathrm{M} \rightarrow \mathrm{M}$ be the flow of a vector field $X$ on $\mathrm{M}$; that is, for all $p \in \mathrm{M}$,

$$
X(p)=\left.\frac{\partial}{\partial t} f_{t}(p)\right|_{t=0} .
$$

The flow for $X_{T \mathrm{M}}$ is given at $z \in T_{p} \mathrm{M}$ by $t \mapsto\left(f_{t}\right)_{*}: T \mathrm{M} \rightarrow T \mathrm{M}$,

$$
X_{T \mathrm{M}}(z)=\left.\frac{\partial}{\partial t}\left(\left(f_{t}\right)_{*} z\right)\right|_{t=0} .
$$

We have

$$
\pi_{*}\left(X_{T \mathrm{M}}(z)\right)=\left.\frac{\partial}{\partial t}\left(\pi\left(\left(f_{t}\right)_{*} z\right)\right)\right|_{t=0}=\left.\frac{\partial}{\partial t}\left(f_{t}(\pi z)\right)\right|_{t=0}=X(p) .
$$

Now, consider the variation $\alpha(t, s)=f_{t}(c(s))$, where $s \mapsto c(s)$ is a path in M with $\dot{c}(0)=z$. Then,

$$
\left.\frac{\partial \alpha}{\partial t}\right|_{t=0}=X(c(s)),\left.\quad \frac{\partial \alpha}{\partial s}\right|_{s=0}=\left(f_{t}\right)_{*}(z) .
$$

Thus, from the definition of the connection map, and the fact that $\nabla$ is torsion-free, that is, $\nabla_{X} Y-\nabla_{Y} X=[X, Y]$, we have

$$
\mathbf{K}\left(X_{T \mathrm{M}}(z)\right)=\left.\nabla_{\frac{\partial \alpha}{\partial t}}\left(\frac{\partial \alpha}{\partial s}\right)\right|_{t=s=0}=\left.\nabla_{\frac{\partial \alpha}{\partial s}}\left(\frac{\partial \alpha}{\partial t}\right)\right|_{t=s=0}=\nabla_{z} X .
$$

Expression (4.2) follows from (2.5), (4.3) and (4.4).

\subsubsection{Expression of $P$ along a Riemannian leaf.}

Let $\gamma$ be a unit-speed geodesic with $\gamma(0)=p$ and let $\mathcal{L}_{\gamma}$ the Riemannian leaf $\mathcal{L}_{\gamma}=\left\{y \dot{\gamma}(x), \mid(x, y) \in \mathbb{R}^{2}\right\} \subset T \mathrm{M}$. We will use, as usual, the parametrization

$$
(t, y) \in \mathbb{R}^{2} \mapsto z=\gamma_{*}\left(y \frac{\partial}{\partial t}\right)=y \dot{\gamma}(t) \in \mathcal{L} \gamma .
$$

We choose an orthonormal basis $\left\{u_{1}, \ldots, u_{n}\right\}$ of $T_{p}$ M. Let $t \rightarrow\left\{J_{1}(t), \ldots, J_{n}(t)\right\} \subset$ $T_{\gamma(t)} \mathrm{M}$ and $t \rightarrow\left\{G_{1}(t), \ldots, G_{n}(t)\right\} \subset T_{\gamma(t)} \mathrm{M}$ be the two sets of Jacobi fields along $\gamma$ defined by the boundary conditions

$$
J_{i}(0)=G_{i}^{\prime}(0)=0, \quad J_{i}^{\prime}(0)=G_{i}(0)=u_{i}, \quad \text { for } 1 \leq i \leq n,
$$

where ' denotes the covariant derivative $\nabla_{\dot{\gamma}(t)}$. Since by hypothesis M has no conjugate points, the set $\left\{J_{1}(t), \ldots, J_{n}(t)\right\}$ is a basis of $T_{\gamma(t)} \mathrm{M}$ for all $t \neq 0$, and hence there are functions $h_{i k}: \mathbb{R} \backslash\{0\} \rightarrow \mathbb{R}$, with $1 \leq i, k \leq n$, such that

$$
G_{i}(t)=\sum_{k=1}^{n} h_{i k}(t) J_{k}(t), 1 \leq i \leq n, \quad \text { for all } t \neq 0 .
$$


Let $\tilde{J}_{i}(t, y)$ and $\tilde{G}_{i}(t, y)$ for $1 \leq i \leq n$ and $(t, y)$ as in (4.5) be the extensions of the Jacobi fields along the Riemannian leaf,

$$
\tilde{J}_{i}(t, y)=\left(J_{i}(t)\right)_{z}^{h}+y\left(J_{i}^{\prime}(t)\right)_{z}^{v}, \quad \tilde{G}_{i}(t, y)=\left(G_{i}(t)\right)_{z}^{h}+y\left(G_{i}^{\prime}(t)\right)_{z}^{v} .
$$

These vector fields are sections of the bundle $\gamma_{*}^{-1}(T(T \mathrm{M}))$ on $\mathbb{R}^{2}$.

By hypothesis $(\mathrm{M}, g)$ have no conjugate points, hence the structure $\mathbf{P}$ is defined on all of TM. Thus, Proposition 2.5 implies that if $J$ is any Jacobi field along the unit-speed geodesic $\gamma$ and $\tilde{J}$ its canonical extension along $\mathcal{L}_{\gamma}$, where we parametrize $\mathcal{L}_{\gamma}$ by $(x, y) \mapsto y \dot{\gamma}(x)$, then

$$
J(s)=0 \Longleftrightarrow[\tilde{J}(x, s-x)]^{+}=0 \forall x \in \mathbb{R} \Longleftrightarrow[\tilde{J}(x, x-s)]^{-}=0 \forall x \in \mathbb{R}
$$

But, since our set of chosen fields $\left\{J_{1}(t), \ldots, J_{n}(t)\right\}$ is linearly independent for all $t \neq 0$, by (4.9) it follows that

$$
\left\{\left[\tilde{J}_{i}(x, y)\right]^{+}, 1 \leq i \leq n\right\}
$$

is a frame for $\gamma_{*}^{-1}\left([T(T \mathrm{M})]^{+}\right)$on $\mathbb{R}^{2} \backslash\{x+y=0\}$; similarly,

$$
\left\{\left[\tilde{J}_{i}(x, y)\right]^{-}, 1 \leq i \leq n\right\}
$$

is a frame for $\gamma_{*}^{-1}\left([T(T \mathrm{M})]^{-}\right)$on $\mathbb{R}^{2} \backslash\{x-y=0\}$.

So, from (4.10) there are functions $h_{i k}^{+}: \mathbb{R}^{2} \backslash\{x+y=0\} \rightarrow \mathbb{R}$ so that

$$
\left[\tilde{G}_{i}(x, y)\right]^{+}=\sum_{k=1}^{n} h_{i k}^{+}(x, y)\left[\tilde{J}_{k}(x, y)\right]^{+}
$$

and from (4.11) there are functions $h_{i k}^{-}: \mathbb{R}^{2} \backslash\{x-y=0\} \rightarrow \mathbb{R}$ so that

$$
\left[\tilde{G}_{i}(x, y)\right]^{-}=\sum_{k=1}^{n} h_{i k}^{-}(x, y)\left[\tilde{J}_{k}(x, y)\right]^{-} .
$$

But from the local description of the extensions of Jacobi in (2.12) it follows that the two-variable functions $h_{i k}^{+}$in (4.12) and $h_{i k}^{-}$in (4.13) satisfy, on their respective domains,

$$
\frac{\partial h_{i k}^{-}}{\partial s^{+}}=0, \quad \frac{\partial h_{i k}^{+}}{\partial s^{-}}=0
$$

hence, there are one-variable functions $f_{i k}^{ \pm}: \mathbb{R} \backslash\{0\} \rightarrow \mathbb{R}$ such that

$$
\left\{\begin{array}{l}
h_{i k}^{+}(x, y)=f_{i k}^{+}(x+y), \text { for } x+y \neq 0, \\
h_{i k}^{-}(x, y)=f_{i k}^{-}(x-y), \text { for } x-y \neq 0 .
\end{array}\right.
$$

Now consider the $h_{i k}^{+}$in each of the two connected regions $\{x+y>0\} \subset \mathbb{R}^{2}$ and $\{x+y<0\} \subset \mathbb{R}^{2}$ and restrict to $y=0$. By (4.12) and the expression of $\mathbf{P}$ along the zero section (2.8) we get, for all $x+y \neq 0$,

$$
h_{i k}^{+}(x, y)=f_{i k}^{+}(x+y)=h_{i k}^{+}(x+y, 0)=h_{i k}(x+y),
$$


with $h_{i k}: \mathbb{R} \backslash\{0\}$ defined by (4.7). Similarly, restricting the $h_{i k}^{-}$defined on each of the connected $\{x-y>0\} \subset \mathbb{R}^{2}$ and $\{x-y<0\} \subset \mathbb{R}^{2}$ to $y=0$, and by (4.13), we get, for all $x-y \neq 0$,

$$
h_{i k}^{-}(x, y)=f_{i k}^{-}(x-y)=h_{i k}^{-}(x-y, 0)=h_{i k}(x-y) .
$$

Thus, on $\mathbb{R}^{2} \backslash\left\{x^{2}-y^{2}=0\right\}$ it holds that

$$
\left[\tilde{G}_{i}(x, y)\right]^{ \pm}=\sum_{k=1}^{n} h_{i k}(x \pm y)\left[\tilde{J}_{k}(x, y)\right]^{ \pm} .
$$

In particular, for $x=0$ and $y \neq 0$, putting $z=y \dot{\gamma}(0)$, and by (4.6) and (4.8), we have

$$
\left[\left(u_{i}\right)_{z}^{h}\right]^{ \pm}=\sum_{k=1}^{n} y h_{i k}( \pm y)\left[\left(u_{k}\right)_{z}^{v}\right]^{ \pm} .
$$

Now, by adding the + and - projections of $\left(u_{i}\right)_{z}^{h}$ in (4.15) we get

$$
\left(u_{i}\right)_{z}^{h}=\sum_{k=1}^{n}\left(\Omega_{\gamma}\right)_{i k}(y)\left(u_{k}\right)_{z}^{v}+\sum_{k=1}^{n}\left(\Delta_{\gamma}\right)_{i k}(y) \mathbf{P}\left(u_{k}\right)_{z}^{v},
$$

where we defined, for all $1 \leq i, k \leq n$ and for all $y \neq 0$,

$$
\begin{aligned}
& \left(\Delta_{\gamma}\right)_{i k}(y) \stackrel{\text { def }}{=} \frac{y}{2}\left(h_{i k}(y)-h_{i k}(-y)\right), \\
& \left(\Omega_{\gamma}\right)_{i k}(y) \stackrel{\text { def }}{=} \frac{y}{2}\left(h_{i k}(y)+h_{i k}(-y)\right) .
\end{aligned}
$$

Remark 4.3. The matrices $\Delta_{\gamma}$ and $\Omega_{\gamma}$ depend not only on the parametrized geodesic $\gamma$ but also on the choice of the basis for $T_{\gamma(0)} \mathrm{M}$. However for simplicity this is not reflected in the notation.

Proposition 4.4. The matrix-valued function $\Delta_{\gamma}$ given by (4.17) is defined for $y=0$. Moreover, for all $y \in \mathbb{R}, \Delta_{\gamma}(y)$ is symmetric and positive-definite. (See Remark 4.5 on $\Omega_{\gamma}$.)

Proof. We note that (4.16) implies that for all $y \neq 0$ the matrix $\Delta_{\gamma}(y)$ is nonsingular, for if there are constants $a_{i}$ so that $\sum_{i=1}^{n} a_{i}\left(\Delta_{\gamma}\right)_{i k}(y)=0$ for a value of $y \neq 0$ and for all $1 \leq k \leq n$, then

$$
\left(\sum_{i=1}^{n} a_{i} u_{i}\right)_{z}^{h}=\sum_{i=1}^{n} \sum_{k=1}^{n} a_{i}\left(\Omega_{\gamma}\right)_{i k}(y)\left(u_{k}\right)_{z}^{v},
$$

and applying $\pi_{*}$ to both sides of (4.18) gives $\sum_{i=1}^{n} a_{i} u_{i}=0$, thus $a_{i}=0$ for all $1 \leq i \leq n$. (See also Remark 4.6.)

We take the limit as $y \rightarrow 0$ on both sides of (4.16) using that

$$
\lim _{y \rightarrow 0}\left(u_{i}\right)_{y \dot{\gamma}(0)}^{h}=\left(u_{i}\right)_{0_{\gamma(0)}}^{h}, \lim _{y \rightarrow 0}\left(u_{i}\right)_{y \dot{\gamma}(0)}^{v}=\left(u_{i}\right)_{0_{\gamma(0)}}^{v}, \lim _{y \rightarrow 0}\left(\mathbf{P}\left(u_{i}\right)_{y \dot{\gamma}(0)}^{v}\right) \stackrel{\dagger}{=}\left(u_{i}\right)_{0_{\gamma(0)}}^{h},
$$

where $(\dagger)$ holds since for all $p \in \mathrm{M}$ and all $u \in T_{p} \mathrm{M}$ we have, by (2.8), $\mathbf{P}(u)_{0_{p}}^{h}=$ $(u)_{0_{p}}^{v}$. Thus we obtain from the comparison of horizontal and vertical lifts in that limit of (4.16), calculated for every $1 \leq i \leq n$, that

$$
\lim _{y \rightarrow 0}\left(\Delta_{\gamma}\right)_{i k}(y)=\delta_{i k}, \quad \lim _{y \rightarrow 0}\left(\Omega_{\gamma}\right)_{i k}(y)=0 .
$$


Since $\lim _{y \rightarrow 0} \Delta_{\gamma}(y)=I$ and $\Delta_{\gamma}(y)$ is non-singular for all $y \neq 0$, we have that $\Delta_{\gamma}(y)$ is positive-definite for all $y \in \mathbb{R}$, as claimed.

We show the symmetry part in the claim. From (4.16), always at $z=y \dot{\gamma}(0)$,

$$
\mathbf{P}\left(u_{i}\right)_{z}^{v}=\sum_{k=1}^{n}\left(\Delta_{\gamma}^{-1}(y)\right)_{i k}\left(u_{k}\right)_{z}^{h}-\sum_{k=1}^{n}\left(\Delta_{\gamma}^{-1}(y) \Omega_{\gamma}(y)\right)_{i k}\left(u_{k}\right)_{z}^{v}
$$

Using this expression back into the equation obtained by applying $\mathbf{P}$ to (4.16) we get

$\mathbf{P}\left(u_{i}\right)_{z}^{h}=\sum_{k=1}^{n}\left(\Omega_{\gamma}(y) \Delta_{\gamma}^{-1}(y)\right)_{i k}\left(u_{k}\right)_{z}^{h}+\sum_{k=1}^{n}\left(\Delta_{\gamma}(y)-\Omega_{\gamma}(y) \Delta_{\gamma}^{-1}(y) \Omega_{\gamma}(y)\right)_{i k}\left(u_{k}\right)_{z}^{v}$.

Finally we compute, for all $1 \leq i, k \leq n$,

$$
d \Theta\left(\mathbf{P}\left(u_{i}\right)_{z}^{v},\left(u_{k}\right)_{z}^{v}\right) \stackrel{(\mathrm{i})}{=}-\left(\Delta_{\gamma}^{-1}(y)\right)_{i k} .
$$

The anti-P-invariance of $d \Theta$ and equalities (4.20) show the symmetry of $\Delta_{\gamma}^{-1}(y)$, hence the symmetry of $\frac{y}{2} \Delta_{\gamma}(y)$, completing the proof of the proposition.

Remark 4.5. In addition,

$$
\begin{aligned}
& d \Theta\left(\mathbf{P}\left(u_{i}\right)_{z}^{v},\left(u_{k}\right)_{z}^{h}\right) \stackrel{(\mathrm{a})}{=}-\left(\Delta_{\gamma}^{-1}(y) \Omega_{\gamma}(y)\right)_{i k}, \\
& d \Theta\left(\mathbf{P}\left(u_{i}\right)_{z}^{h},\left(u_{k}\right)_{z}^{v}\right) \stackrel{(\mathrm{b})}{=}-\left(\Omega_{\gamma}(y) \Delta_{\gamma}^{-1}(y)\right)_{i k} \\
& d \Theta\left(\mathbf{P}\left(u_{i}\right)_{z}^{h},\left(u_{k}\right)_{z}^{h}\right)=\left(\Delta_{\gamma}(y)-\Omega_{\gamma}(y) \Delta_{\gamma}^{-1}(y) \Omega_{\gamma}(y)\right)_{i k} .
\end{aligned}
$$

Then, from (a) and (b), the anti-P-invariance of $d \Theta$, the symmetry of $\Delta_{\gamma}(y)$ shown in Proposition 4.4, and the right-hand side equation in (4.19), it follows that, for all $y \in \mathbb{R}, \Omega_{\gamma}(y)$ is well defined and symmetric.

Remark 4.6. Another way to see that the matrix $\Delta_{\gamma}(y)$ is non-singular for $y \neq 0$ : Let $a_{i} \in \mathbb{R}$ and $0 \neq y \in \mathbb{R}$ such that $0=\sum_{i=1}^{n} a_{i}\left(h_{i k}(y)-h_{i k}(-y)\right)$. Then the Jacobi vector field $J(t) \stackrel{\text { def }}{=} \sum_{k=1}^{n} a_{i} G_{i}(t)-\sum_{i, k=1}^{n} a_{i} h_{i k}(-y) J_{k}(t)$ vanishes for $t=y$ and for $t=-y$. Since $\mathrm{M}$ has no conjugate points, in particular $J(0)=$ $\sum_{i=1}^{n} a_{i} u_{i}=0$; hence $a_{i}=0, \forall i$.

Proposition 4.7. The matrix-valued function $y \rightarrow y \Delta_{\gamma}^{-1}(y)$ is increasing, in the sense of matrices, on $(0, \infty)$. That is, for all $y$ and $y_{1}$ in $\mathbb{R}$,

$$
0<y_{1}<y \Longrightarrow\left(y \Delta_{\gamma}^{-1}(y)-y_{1} \Delta_{\gamma}^{-1}\left(y_{1}\right)\right) \text { is positive-definite. }
$$

(It is also increasing on $(-\infty, 0)$, being an odd function.)

Proof. Along the geodesic $\gamma$, given two Jacobi fields $A$ and $B$, we denote by $W(A, B)$ their Wronskian,

$$
W(A, B)=g\left(A^{\prime}(t), B(t)\right)-g\left(A(t), B^{\prime}(t)\right) \stackrel{(*)}{=} g\left(A^{\prime}(0), B(0)\right)-g\left(A(0), B^{\prime}(0)\right),
$$

with equality $(*)$ valid for all $t \in \mathbb{R}$, a standard consequence of the equation defining Jacobi fields. In particular, for the Jacobi fields $G_{i}$ and $J_{i}$ defined earlier by their conditions at $t=0$ (4.6) we have, for all $1 \leq i, k \leq n$ and $\delta_{i k}$ the Kroenecker delta,

$$
W\left(G_{i}, G_{k}\right)=W\left(J_{i}, J_{k}\right)=0, \quad W\left(G_{i}, J_{k}\right)=-\delta_{i k} .
$$


For all $t \neq 0,2 t^{-1} \Delta_{\gamma}(t)=h(t)-h(-t)$, where $h(t)$ is the matrix defined by $G_{i}(t)=\sum_{k=1}^{n} h_{i k}(t) J_{k}(t)$, and thus, we calculate, for $t \neq 0$,

$$
\begin{aligned}
-\delta_{i l} & =W\left(G_{i}, J_{l}\right) \\
& =\sum_{k=1}^{n} h_{i k}^{\prime}(t) g\left(J_{k}(t), J_{l}(t)\right)+\sum_{k=1}^{n} h_{i k}(t) W\left(J_{k}, J_{l}\right) \\
& \stackrel{(\#)}{=} \sum_{k=1}^{n} h_{i k}^{\prime}(t) g\left(J_{k}(t), J_{l}(t)\right) .
\end{aligned}
$$

But, since $\mathrm{M}$ is assumed to have no conjugate points, the matrix with entries $g\left(J_{k}(t), J_{l}(t)\right)$ is positive-definite for $t \neq 0$. Moreover, (\#) shows that the negative of its inverse matrix is just $h^{\prime}(t)$, and hence $h^{\prime}(t)$ is negative-definite for all $t \neq 0$. It follows that given a constant $y_{1}>0$ we have for $y>y_{1}$,

$$
\begin{aligned}
2 y^{-1} \Delta_{\gamma}(y) & =h(y)-h(-y) \\
& =h\left(y_{1}\right)-h\left(-y_{1}\right)+\int_{-y}^{-y_{1}} h^{\prime}(t) d t+\int_{y_{1}}^{y} h^{\prime}(t) d t<2 y_{1}^{-1} \Delta_{\gamma}\left(y_{1}\right),
\end{aligned}
$$

and, inverting, $y \Delta_{\gamma}^{-1}(y)>y_{1} \Delta_{\gamma}^{-1}\left(y_{1}\right)$ for all $y>y_{1}>0$, as claimed.

4.0.3. Example: Let $(\mathrm{M}, g)$ be a symmetric space of non-positive curvature of dimension $n$. Let $\gamma$ be a unit-speed geodesic with $\gamma(0)=p$. Since the curvature tensor $\mathbf{R}_{\mathrm{M}}$ of $\mathrm{M}$ is parallel (see [12]), there is a parallel frame $\left\{E_{1}, \ldots, E_{n}\right\}$ along $\gamma$ with $g\left(E_{i}(t), E_{j}(t)\right)=\delta_{i j}$ and $\mathbf{R}_{\mathrm{M}}\left(E_{i}(t), \dot{\gamma}(t)\right) \dot{\gamma}(t)=-\lambda_{i}^{2} E_{i}(t), 1 \leq i, j \leq n$, for all $t \in \mathbb{R}$. Let $J_{i}(t)$ be the Jacobi fields $J_{i}(t)=\lambda_{i}^{-1} \sinh \left(t \lambda_{i}\right) E_{i}(t)$ if $\lambda_{i}>0$ or $J_{i}(t)=t E_{i}(t)$ if $\lambda_{i}=0$. Also, let $G_{i}(t)$ be the Jacobi fields $G_{i}(t)=\cosh \left(t \lambda_{i}\right) E_{i}(t)$ if $\lambda_{i}>0$ or $G_{i}(t)=E_{i}(t)$ if $\lambda_{i}=0$. We have, with the notation as above, for $t \neq 0$,

$$
h_{i k}(t)=\left\{\begin{array}{l}
\delta_{i k} \lambda_{i} \operatorname{coth}\left(t \lambda_{i}\right) \text { if } \lambda_{i}>0 \\
\delta_{i k} t^{-1} \text { if } \lambda_{i}=0
\end{array}\right.
$$

Thus, the matrices (4.17) constructed for $\gamma$ and for the selected orthonormal basis for $T_{p} \mathrm{M}$ are given by

$$
\left(\Delta_{\gamma}\right)_{i k}(y)=\left\{\begin{array}{l}
y \lambda_{i} \operatorname{coth}\left(y \lambda_{i}\right) \text { if } \lambda_{i}>0, \quad\left(\Omega_{\gamma}\right)_{i k}=0 . \\
1 \text { if } \lambda_{i}=0,
\end{array}\right.
$$

So, for $0 \neq z \in T \mathrm{M}$ and $\left\{v_{i}\right\}_{1 \leq i \leq n}$ a basis of $T_{\pi(z)} \mathbf{M}$ with $\mathbf{R}_{\mathrm{M}}\left(v_{i}, z\right) z=-\lambda_{i}^{2}\|z\|^{2} v_{i}$,

$$
\mathbf{P}\left(v_{i}\right)_{z}^{h}=f_{i}(z)\left(v_{i}\right)_{z}^{v}
$$

where

$$
f_{i}(z)=\left\{\begin{array}{l}
\lambda_{i}\|z\| \operatorname{coth}\left(\lambda_{i}\|z\|\right) \text { if } \lambda_{i}>0 \\
1 \text { if } \lambda_{i}=0
\end{array}\right.
$$

which, together with (2.8) for $z=0$, describes $\mathbf{P}$ on $T \mathrm{M}$. 
4.0.4. Proof of Theorem 4.1. (See the statement following (4.1).)

Proof. Let $X: \mathrm{M} \rightarrow T \mathrm{M}$ be a vector field on $\mathrm{M}$ and $X_{T \mathrm{M}}$ the vector field on $T \mathrm{M}$ induced by the tangent maps of the flow of $X$ on M, so that by Proposition 4.2 for any $z \in T_{p} \mathrm{M}, X_{T \mathrm{M}}(z)=(X(p))_{z}^{h}+\left(\nabla_{z} X\right)_{z}^{v}$.

Let us take $z=a X(p) \in \mathcal{S}_{X} \cap T_{p} \mathrm{M}$ where $0 \neq a \in \mathbb{R}$ is a constant. Then we have

$$
\begin{aligned}
& X_{T \mathrm{M}}(a X(p))=(X(p))_{a X(p)}^{h}+\left(\nabla_{a X(p)} X\right)_{a X(p)}^{v} \\
&=\left.\left(\left(a^{-1} z\right)_{z}^{h}+\left(\nabla_{z} X\right)_{z}^{v}\right)\right|_{z=a X(p)}, \\
&\left.\mathbf{P} X_{T \mathrm{M}}(a X(p)) \stackrel{(*)}{=}\left(\left(a^{-1} z\right)_{z}^{v}+\mathbf{P}\left(\nabla_{z} X\right)_{z}^{v}\right)\right|_{z=a X(p)},
\end{aligned}
$$

where $(*)$ uses that $\mathbf{P}\left(a^{-1} z\right)_{z}^{h}=\left(a^{-1} z\right)_{z}^{v}$, and thus,

$\overbrace{\left.d \Theta\left(\mathbf{P} X_{T \mathrm{M}}(z), X_{T \mathrm{M}}(z)\right)\right|_{z=a X(p)}}^{(\mathrm{I})}=\overbrace{\|X(p)\|^{2}}^{(\mathrm{II})}+\overbrace{\left.d \Theta\left(\mathbf{P}\left(\nabla_{z} X\right)_{z}^{v},\left(\nabla_{z} X\right)_{z}^{v}\right)\right|_{z=a X(p)}}^{(\mathrm{III})}$.

Above we used

$$
d \Theta\left((X(p))_{z}^{v},\left(\nabla_{z} X\right)_{z}^{v}\right)=0
$$

true by (2.4), since both arguments are vertical; also we used

$$
\left.d \Theta\left(\mathbf{P}\left(\nabla_{z} X\right)_{z}^{v},(X(p))_{z}^{h}\right)\right|_{z=a X(p)}=-\left.d \Theta\left(\left(\nabla_{z} X\right)_{z}^{v},(X(p))_{z}^{v}\right)\right|_{z=a X(p)}=0,
$$

which follows from the anti-P-invariance of $d \Theta$ and, again, $\mathbf{P}\left(a^{-1} z\right)_{z}^{h}=\left(a^{-1} z\right)_{z}^{v}$.

The term (III) is calculated using an orthonormal basis of $T_{p} \mathrm{M},\left\{u_{i}, \ldots, u_{n}\right\}$ :

$$
\begin{aligned}
(\mathrm{III}) & =a^{2} \sum_{i=1}^{n} \sum_{j=1}^{n} g\left(\nabla_{X(p)} X, u_{i}\right) g\left(\nabla_{X(p)} X, u_{j}\right) d \Theta\left(\mathbf{P}\left(u_{i}\right)_{a X(p)}^{v},\left(u_{j}\right)_{a X(p)}^{v}\right) \\
& =-a^{2} \sum_{i=1}^{n} \sum_{j=1}^{n} g\left(\nabla_{X(p)} X, u_{i}\right) g\left(\nabla_{X(p)} X, u_{j}\right)\left(\Delta_{\gamma}^{-1}\right)_{i j}(|a|\|X(p)\|),
\end{aligned}
$$

where the second equality, in terms of the matrix-valued function $y \rightarrow \Delta_{\gamma}^{-1}(y)$ constructed for the unit-speed geodesic $\gamma$ with initial condition $\dot{\gamma}(0)=X(p) /\|X(p)\|$ and the chosen basis of $T_{p} \mathrm{M}$, uses that for $z=y \dot{\gamma}(0)$,

$$
d \Theta\left(\mathbf{P}\left(u_{i}\right)_{z}^{v},\left(u_{j}\right)_{z}^{v}\right)=-\left(\Delta_{\gamma}^{-1}\right)_{i j}(\|z\|)
$$

by (4.20) and $\left(\Delta_{\gamma}^{-1}\right)(-y)=\left(\Delta_{\gamma}^{-1}\right)(y)$.

Now, from Proposition 4.7, for all $|a|>1$ we have the matrix inequality

$$
|a| \Delta_{\gamma}^{-1}(|a|\|X(p)\|)>\Delta_{\gamma}^{-1}(\|X(p)\|),
$$

and thus

$$
\text { (III) }<-|a| \sum_{i=1}^{n} \sum_{j=1}^{n} g\left(\nabla_{X(p)} X, u_{i}\right) g\left(\nabla_{X(p)} X, u_{j}\right)\left(\Delta_{\gamma}^{-1}\right)_{i j}(\|X(p)\|) \text { for }|a|>1 .
$$

But by Proposition $4.4 \Delta_{\gamma}^{-1}(\|X(p)\|)$ is positive-definite, and hence, we have that $\nabla_{X(p)} X \neq 0$ if and only if by taking $|a|>>1$ we can make (III) $<-\|X(p)\|^{2}=$ - (II) so that $(\mathrm{I})<0$.

It follows that

$$
\nabla_{X(p)} X=0 \Longleftrightarrow d \Theta\left(\mathbf{P} X_{T \mathrm{M}}, X_{T \mathrm{M}}\right)=\mathbf{G}_{\mathbf{P}}\left(X_{T \mathrm{M}}, X_{T \mathrm{M}}\right) \geq 0 \text { on } \mathbb{R} X(p) .
$$


The proof of Theorem 4.1 is completed by applying the argument above to each point $p \in \mathrm{M}$.

\section{RESTRICTIONS ON TANGENTIALly POSITIVE ACTIONS}

In this section we derive from Theorem 4.1 and the propositions used for its proof some restrictions on isometric actions, on conjugate-point free manifolds (M,g), tangentially positive in two canonical subsets of $T \mathrm{M}$ canonically associated to the $G$-action, namely, $\mu_{G}^{-1}(0) \subset T \mathrm{M}$ and

$$
\mathfrak{G}^{\mathrm{M}} \stackrel{\text { def }}{=}\left\{\xi_{\mathrm{M}}(p) \mid \xi \in \mathfrak{G}, p \in \mathrm{M}\right\} \subset T \mathrm{M} .
$$

We relate tangentially positive actions on $\mathfrak{G}^{\mathrm{M}}$ with curvature in Proposition 5.1, and on $\mu_{G}{ }^{-1}(0)$ to fixed points in Proposition 5.8. Of course, there is no apriori assumption on the freeness of the $G$-action, as this is necessary for our applications to induced actions in the factors of a Riemannian product. (If the action is free $\mathfrak{G}^{\mathrm{M}}$ is just the total space of the vertical bundle for the principal bundle $f: \mathrm{M} \rightarrow \mathrm{M} / G$.)

If we identify $\mathrm{M}$ with the zero section of $T \mathrm{M}$,

$$
\mathrm{M}=\mu_{G}{ }^{-1}(0) \cap \mathfrak{G}^{\mathrm{M}},
$$

and, in general, the tangentially positivity on these two sets is independent. For instance, the $\mathbb{R}$-action in $\mathrm{H}^{n}$ coming up in (7.6) is not tangentially positive in all of $\mathfrak{G}^{\mathrm{H}^{n}}$, but it is tangentially positive in $\mu_{G}{ }^{-1}(0)$ as shown in the paragraphs after (17.6). Also note that since

$$
H \subset G \Longrightarrow\left\{\begin{array}{l}
\mathfrak{H}^{\mathrm{M}} \subset \mathfrak{G}^{\mathrm{M}} \\
\mu_{H}^{-1}(0) \supset \mu_{G}^{-1}(0)
\end{array}\right.
$$

tangentially positivity on these sets behaves differently when considering of action by subgroups.

We will use the following notation. Given a vector field $X$ on $\mathrm{M}$ set

$$
\operatorname{Zero}(X)=\{p \in \mathrm{M} \mid X(p)=0\} \subset \mathrm{M} .
$$

Let $G$ be a group acting on M. We put

$$
\text { Fix }(G) \stackrel{\text { def }}{=}\{x \in \mathrm{M} \mid g \cdot x=x \forall g \in G\} \subset \mathrm{M},
$$

and given $p \in \mathrm{M}$

$$
\operatorname{Isotr}(p)=\{g \in G \mid g \cdot p=p\} \subset G \text {. }
$$

5.0.5. Tangential positivity on $\mathfrak{G}^{\mathrm{M}}$ and curvature.

Proposition 5.1. Let $(\mathrm{M}, g)$ be any complete Riemannian manifold with no conjugate points. Let $G$ act isometrically on $\mathrm{M}$, and tangentially positively on $\mathfrak{G}^{\mathrm{M}}$. Given $\xi \in \mathfrak{G}$, then Zero $\left(\xi_{\mathrm{M}}\right)$ equals $\emptyset$ or $\mathrm{M}$. Moreover, if $\xi_{\mathrm{M}}$ is not identically zero, the sectional curvature of any two-plane containing $\xi_{\mathrm{M}}$ is non-negative.

Remark 5.2. Thus, under the assumptions in Proposition 5.1] it follows that $\mathfrak{G}^{\mathrm{M}}$ is a vector sub-bundle of $T \mathrm{M}$ of $\operatorname{rank} \nu, \nu \stackrel{\text { def }}{=} \operatorname{dim}\left\{\xi_{\mathrm{M}}(p), \xi \in \mathfrak{G}\right\}$ for any $p \in \mathrm{M}$. 
Proof. By Theorem 4.1 the Killing vector fields induced by the action are 'selfparallel',

$$
\nabla_{\xi_{\mathrm{M}}} \xi_{\mathrm{M}}=0 \text { on } \mathrm{M}, \text { for all } \xi \in \mathfrak{G} .
$$

Then, $f=\left\|\xi_{\mathrm{M}}\right\|^{2}$ is constant throughout M, for, using the skew-symmetry of $\nabla \xi_{\mathrm{M}}$, for all $v \in T \mathrm{M}, d f(v)=2 g\left(\nabla_{v} \xi_{\mathrm{M}}, \xi_{\mathrm{M}}\right)=-2 g\left(\nabla_{\xi_{\mathrm{M}}} \xi_{\mathrm{M}}, v\right)=0$. Thus, given $\xi \in \mathfrak{G}$ either $\xi_{\mathrm{M}}$ is identically zero on $\mathrm{M}$ or $\xi_{\mathrm{M}}(p) \neq 0$ for all $p \in \mathrm{M}$ as claimed.

Let $\xi_{\mathrm{M}}$ not be identically zero, and let $X$ be a vector field on M. Concerning the sectional curvature of the plane spanned by $\xi_{\mathrm{M}}$ and $X$ when both are non-zero, note that the curvature operator on $\mathrm{M}, \mathbf{R}_{\mathrm{M}}(X, Y)=\left[\nabla_{X}, \nabla_{Y}\right]-\nabla_{[X, Y]}$, satisfies, if $\xi_{\mathrm{M}}$ is a self-parallel Killing vector field,

$$
g\left(\mathbf{R}_{\mathrm{M}}\left(X, \xi_{\mathrm{M}}\right) \xi_{\mathrm{M}}, X\right)=\left\|\nabla_{X} \xi_{\mathrm{M}}\right\|^{2} \geq 0 .
$$

This formula is shown using (5.7) together with the fact that $\nabla \xi_{\mathrm{M}}$ is skew-symmetric and that $\nabla$ torsion-free. (For a proof of (5.8) see in [1] the proof of Proposition 6.1, formula 6.3.)

Remark 5.3. Recall that the rank of a geodesic $\gamma$ is the number of linearly independent parallel Jacobi fields along the geodesic, including its velocity $\dot{\gamma}$. It cannot exceed the dimension of $\mathrm{M}$, as Jacobi fields are solutions of a second order linear equation. Also, rank $\mathrm{M}$ is the minimum of that number taken over all geodesics.

Corollary 5.4. Let $(\mathrm{M}, g)$ and $G$ be as in Proposition 5.1 acting tangentially positively on $\mathfrak{G}^{\mathrm{M}}$, and assume $G$ connected. Then,

1) if $\mathrm{M}$ is flat $G$ acts by translations;

2) if $\exists p \in \mathrm{M}$ where the Ricci curvature tensor of $\mathrm{M}$ is negative-definite, $G$ acts trivially on $\mathrm{M}$;

3) if $\mathrm{M}$ is non-positively curved, $\operatorname{dim} \mathrm{M}=n \geq \operatorname{rank} \mathrm{M} \geq \min \{\nu+1, n\}$.

Proof. Part 1: If M is flat, by (5.8), $0=\left|\nabla_{u} \xi_{\mathrm{M}}\right|^{2} \geq 0$ for all $p \in \mathrm{M}$ and all $u \in T_{p} \mathrm{M}$, hence $\nabla \xi_{\mathrm{M}}=0$ for all $\xi \in \mathfrak{G}$.

Part 2: Let $\xi \in \mathfrak{G}$ with $\xi_{\mathrm{M}}$ not identically zero. By Proposition $5.1 \xi_{\mathrm{M}}(p) \neq 0$ for all $p \in \mathrm{M}$. Then, by (5.8), $g\left(\mathbf{R}_{\mathrm{M}}\left(u, \xi_{\mathrm{M}}(p)\right) \xi_{\mathrm{M}}(p), u\right) \geq 0$, and thus the Ricci tensor evaluated at $\xi_{\mathrm{M}}(p)$ is non-negative. So, if there is $p \in \mathrm{M}$ where the Ricci tensor is negative-definite, we must have $\xi_{\mathrm{M}}$ identically zero.

Part 3: Let $\gamma$ be a unit-speed rank-minimizing geodesic, i.e., rank of $\gamma=$ rank $\mathrm{M}$, starting at $p$. There are two possibilities:

a) $\dot{\gamma}(0) \notin\left\{\xi_{\mathrm{M}}(p) \mid \xi \in \mathfrak{G}\right\}$, and b) $\dot{\gamma}(0) \in\left\{\xi_{\mathrm{M}}(p) \mid \xi \in \mathfrak{G}\right\}$.

Case a): Let $\xi^{i} \in \mathfrak{G}$ be such that $\left\{\xi_{\mathrm{M}}^{i}(p), 1 \leq i \leq \nu\right\}$ is a basis for $\left\{\xi_{\mathrm{M}}(p) \mid \xi \in \mathfrak{G}\right\}$. (Recall that $\nu$ is independent of $p$. See Remark 5.2.) Then,

$$
\left\{J^{0} \stackrel{\text { def }}{=} \dot{\gamma}, J^{1} \stackrel{\text { def }}{=} \xi_{\mathrm{M}}^{1} \circ \gamma, \ldots, J^{\nu} \stackrel{\text { def }}{=} \xi_{\mathrm{M}}^{\nu} \circ \gamma\right\}
$$

are $\nu+1$ linearly independent Jacobi fields along $\gamma$. In light of (5.8), the curvature assumption and the definition of the $J_{i}$ 's,

$$
0 \geq g\left(\mathbf{R}_{\mathrm{M}}\left(J^{i}(t), \dot{\gamma}(t)\right) \dot{\gamma}(t), J^{i}(t)\right)=\left\|\nabla_{\dot{\gamma}(t)} J^{i}(t)\right\|^{2} \geq 0 .
$$

So, $\left\{J^{i}, \quad 0 \leq i \leq \nu\right\}$ is a set of parallel, linearly independent Jacobi fields along $\gamma$, hence

$$
\nu+1 \leq \operatorname{rank} \gamma=\operatorname{rank} \mathrm{M}
$$


Case b): In this situation, there is a $\xi \in \mathfrak{G}$ such that $\dot{\gamma}(0)=\xi_{\mathrm{M}}(p)$. Since $\nabla_{\xi_{\mathrm{M}}} \xi_{\mathrm{M}}=0$ on M, the geodesic $\gamma$ is the trajectory through $p$ of the action that corresponds to $\xi$, i.e., $\gamma(t)=\exp (t \xi) \cdot p$, and $\dot{\gamma}(t)=\xi_{\mathrm{M}}(\gamma(t))$ for all $t \in \mathbb{R}$. Also, by (5.8) and the non-positivity of the sectional curvatures, $g\left(\mathbf{R}\left(E_{i}(t), \dot{\gamma}(t)\right) \dot{\gamma}(t), E_{i}(t)\right)=0$, $1 \leq i \leq n$, for any frame $\left\{E_{1}, \ldots, E_{n}\right\}$ along $\gamma$, which implies, due to the symmetry of the curvature, that $g\left(\mathbf{R}\left(E_{i}(t), \dot{\gamma}(t)\right) \dot{\gamma}(t), E_{j}(t)\right)=0$ for all $1 \leq i, j \leq n$, and thus $\mathbf{R}\left(E_{i}(t), \dot{\gamma}(t)\right) \dot{\gamma}=0$ for $1 \leq i \leq n$. Now, by taking the frame to be parallel along $\gamma$, i.e, for all $1 \leq i \leq n, \nabla_{\dot{\gamma}(t)} E_{i}=0$ for all $t \in \mathbb{R}$, we have $\nabla_{\dot{\gamma}(t)}^{2} E_{i}+\mathbf{R}_{\mathrm{M}}\left(E_{i}(t), \dot{\gamma}(t)\right) \dot{\gamma}(t)=0$, and thus each $E_{i}$ is a parallel Jacobi field along $\gamma$. In light of this and Remark 5.3 it follows that

$$
\operatorname{rank} \gamma=n \text {. }
$$

From (5.9) and (5.10), $\operatorname{rank} \mathrm{M} \geq \min \{\nu+1, n\}$.

Remark 5.5. If $G$ is not connected use the reasoning in Corollary 3.3 to derive the corresponding statements. Part 3 implies, for example, that if $\mathrm{M}$ is non-positively curved but not flat, then the codimension of the fibers of any isometric action on $\mathrm{M}$ which is tangentially positive on $\mathfrak{G}^{\mathrm{M}}$ must be at least 2 .

5.0.6. Tangential positivity in $\mu_{G}^{-1}(0)$ and fixed points in $\mathrm{M}$.

It is well known that for any isometric action of a connected group on a connected manifold with no conjugate points, Fix $(G)$ as in (5.5) is a connected totally geodesic submanifold. (See [10.) In particular, if $\zeta_{\mathrm{M}}$ is an infinitesimal action vector field on $M$,

$$
\operatorname{Zero}\left(\zeta_{\mathrm{M}}\right)=\operatorname{Fix}(\{t \mapsto \exp t \xi, t \in \mathbb{R}\})
$$

is a connected totally geodesic manifold. We now derive further restrictions on their presence when $G$ acts tangentially positively in $\mu_{G}^{-1}(0)$.

Proposition 5.6. Let $(\mathrm{M}, g)$ be complete with no conjugate points. Let $G$ act isometrically on $\mathrm{M}$ tangentially positively on $\mu_{G}^{-1}(0) \subset T \mathrm{M}$. Then any infinitesimal action vector field $\zeta_{\mathrm{M}}$ satisfies the transversality condition

$$
\forall p \in Z \operatorname{Zero}\left(\zeta_{\mathrm{M}}\right), \quad T_{p}\left(Z \operatorname{ero}\left(\zeta_{\mathrm{M}}\right)\right)+\mathfrak{G}_{p}^{\mathrm{M}}=T_{p} \mathrm{M},
$$

where the sum is not necessarily direct.

Corollary 5.7. Consequently, the orbits of the G-action on $\mathrm{M}$ have all the same dimension. (In particular, Fix $\left(G_{0}\right)=\emptyset$ or $\mathrm{M}$ for $G_{0}$, the connected component of G.)

Proof. First we prove Proposition [5.6. If $\zeta_{\mathrm{M}}$ is identically zero $\operatorname{Zero}\left(\zeta_{\mathrm{M}}\right)=\mathrm{M}$, and (5.12) is then true. So, we assume that there is $\zeta_{\mathrm{M}}$ not identically zero and $p$ in Zero $\left(\zeta_{\mathrm{M}}\right)$ so that the condition (5.12) is not met at $p$, while the action is tangentially positive in $\mu_{G}^{-1}(0)$, and derive a contradiction.

Since, by definition of $\mu_{G}, T_{p} M=\mathfrak{G}_{p}^{\mathrm{M}} \oplus\left(\mu_{G}^{-1}(0) \cap T_{p} \mathrm{M}\right)$, if (5.12) fails at $p$ it follows that

$$
\left(\mu_{G}^{-1}(0) \cap T_{p} \mathrm{M}\right) \not \subset T_{p}\left(Z \operatorname{ero}\left(\zeta_{\mathrm{M}}\right)\right) .
$$

Let $z \in\left(\mu_{G}^{-1}(0) \cap T_{p} \mathrm{M}\right) \backslash T_{p}\left(Z \operatorname{Zero}\left(\zeta_{\mathrm{M}}\right)\right) \neq \emptyset$ by (15.13) . Note that $z \neq 0=0_{p} \simeq p$, and since $\zeta_{\mathrm{M}}$ vanishes at $p, \nabla_{z} \zeta_{\mathrm{M}} \neq 0$. For, otherwise, $\zeta_{\mathrm{M}}$ restricted to the geodesic $\gamma_{z}$ with initial conditions $\|z\| \dot{\gamma}_{z}(0)=z$ would be a Jacobi field $J=\zeta_{\mathrm{M}} \circ \gamma_{z}$ along $\gamma_{z}$ with $J(0)=\zeta_{\mathrm{M}}(p)=0=\nabla_{z} \zeta_{\mathrm{M}}=\|z\| J^{\prime}(0)$, hence identically zero along $\gamma_{z}$, 
which forces $z$ to be in $T_{p}\left(\operatorname{Zero}\left(\zeta_{\mathrm{M}}\right)\right)$, contrary to the way it was picked. Thus, the failure of (5.12) implies that

$$
\exists z \in \mu_{G}^{-1}(0) \cap T_{p} \mathrm{M} \mid \nabla_{z} \zeta_{\mathrm{M}} \neq 0 .
$$

Pick an orthonormal basis $\left\{u_{1}, \ldots, u_{n}\right\}$ of $T_{p}$ M. Let $z$ be as in (5.14). Since $\zeta_{\mathrm{M}}(p)=0$ and $\nabla_{z} \zeta_{\mathrm{M}} \neq 0$

$$
0 \neq \zeta_{T \mathrm{M}}(z)=\left(\zeta_{\mathrm{M}}(p)\right)_{z}^{h}+\left(\nabla_{z} \zeta_{\mathrm{M}}\right)_{z}^{v}=\left(\nabla_{z} \zeta_{\mathrm{M}}\right)_{z}^{v}=\sum_{i=1}^{n} g\left(\nabla_{z} \zeta_{\mathrm{M}}, u_{i}\right)\left(u_{i}\right)_{z}^{v}
$$

and thus,

$$
\begin{aligned}
d \Theta\left(\mathbf{P} \zeta_{T \mathrm{M}}(z), \zeta_{T \mathrm{M}}(z)\right) & =\sum_{i=1}^{n} \sum_{j=1}^{n} g\left(\nabla_{z} \zeta_{\mathrm{M}}, u_{i}\right) g\left(\nabla_{z} \zeta_{\mathrm{M}}, u_{j}\right) d \Theta\left(\mathbf{P}\left(u_{i}\right)_{z}^{v},\left(u_{j}\right)_{z}^{v}\right) \\
& \stackrel{(1)}{=}-\frac{2}{\|z\|} \sum_{i=1}^{n} \sum_{j=1}^{n} g\left(\nabla_{z} \zeta_{\mathrm{M}}, u_{i}\right) g\left(\nabla_{z} \zeta_{\mathrm{M}}, u_{j}\right)\left(\Delta_{\gamma_{z}}\right)_{i j}^{-1}(\|z\|) \\
& \stackrel{(2)}{<} 0,
\end{aligned}
$$

where the matrix $\left(\Delta_{\gamma_{z}}\right)(\|z\|)$ is defined as in (4.17) and constructed along the unitspeed geodesic $\gamma_{z}$ and with respect to the chosen orthonormal basis. The equality (1) follows from the equality (i) in (4.20), and inequality (2) from Proposition (4.4) which implies that $\left(\Delta_{\gamma_{z}}\right)^{-1}(\|z\|)$ is positive-definite.

But since $z \in \mu_{G}^{-1}(0) \cap T_{p} \mathrm{M}$ we have $d \Theta\left(\mathbf{P} \zeta_{T \mathrm{M}}(z), \zeta_{T \mathrm{M}}(z)\right)>0$, since by hypothesis the action is tangentially positive in $\mu_{G}{ }^{-1}(0)$, and we get a contradiction. The proposition is proved.

Now we prove Corollary 5.7 For $p \in \mathrm{M}$ consider the Lie subgroup $\operatorname{Isotr}(p) \subset G$ as in (5.6). Any $\zeta \in \mathfrak{G}$ belongs to its Lie Algebra $\mathfrak{I}_{p} \subset \mathfrak{G}$ if and only if $\zeta_{\mathrm{M}}(p)=0$. Thus, if $\zeta \in \mathfrak{I}_{p}$, since the action is tangentially positive in $\mu_{G}^{-1}(0)$, as shown by the expression (5.16) we must have $\nabla_{z} \zeta_{\mathrm{M}}=0$ for all $z$ in $\mu_{G}^{-1}(0) \cap T_{p} \mathrm{M}$, that is, for all $z g$-orthogonal to $\mathfrak{G}_{p}^{\mathrm{M}}=T_{p}(G \cdot p)$. So, for each $0 \neq z \in \mu_{G}^{-1}(0) \cap T_{p} \mathrm{M}$, if $\gamma_{z}$ is the unit-speed geodesic with initial velocity $\dot{\gamma}_{z}(0)=z /\|z\|$, the Jacobi field $\zeta_{\mathrm{M}} \circ \gamma_{z}$ vanishes for all $t \in \mathbb{R}$, which means that $\zeta \in \mathfrak{I}_{\gamma_{z}(t)}$ for all $t$. It follows that $\mathfrak{I}_{p} \subset \mathfrak{I}_{\gamma_{z}(t)}$ for all $t \in \mathbb{R}$ and all $z \in \mu_{G}^{-1}(0) \cap T_{p} \mathrm{M}$, implying

(5.17) $\operatorname{dim} \operatorname{Isotr}(p) \leq \operatorname{dim} \operatorname{Isotr}\left(\gamma_{z}(t)\right), \quad t \in \mathbb{R}, \forall z g$-orthogonal to $T_{p}(G \cdot p)$.

But for all $g \in G, \operatorname{Isotr}(g \cdot p)=g \operatorname{Isotr}(g \cdot p) g^{-1}$, hence

$$
\operatorname{dim} \operatorname{Isotr}(p)=\operatorname{dim} \operatorname{Isotr}(g \cdot p) .
$$

It thus follows from (5.17), using the exponential map in $\mathrm{M}$ restricted to the normal bundle to the orbit, that there is an open set $\mathcal{U} \subset \mathrm{M}$ containing $p$ such that $\operatorname{dim} \operatorname{Isotr}(p) \leq \operatorname{dim} \operatorname{Isotr}(x)$ for all $x \in \mathcal{U}$, that is,

$$
\operatorname{dim} T_{p}(G \cdot p) \geq \operatorname{dim} T_{x}(G \cdot x), \forall x \in \mathcal{U} .
$$

But, if we took $p$ in (5.18) as a point in an orbit with minimal dimension ('singular orbit'), it would follow that the set of orbits of maximal dimension ('principal orbits') is not dense in $\mathrm{M}$, which is well known not to be the case. Thus all orbits have the same dimension. 
The connected component $G_{0} \subset G$ is a normal Lie subgroup of $G$. The statement in Corollary 5.7 concerning $G_{0}$ generalizes for arbitrary normal Lie subgroups as follows.

Proposition 5.8. Let $(\mathrm{M}, g)$ be complete connected with no conjugate points. Let a Lie group $G$ act isometrically on $\mathrm{M}$ tangentially positively on $\mu_{G}^{-1}(0) \subset T \mathrm{M}$.

0) If $H \subset G$ is a normal Lie subgroup of $G$, then Fix $(H)$ equals either a set of isolated points (possibly empty), or M.

Consequently, for $G$ so acting,

i) If $H \subset G$ is normal and connected it either acts freely, or trivially on M. In the latter case $\mu_{G / H}^{-1}(0)=\mu_{G}^{-1}(0)$ and $G / H$ acts on $\mathrm{M}$ tangentially positively on $\mu_{G}^{-1}(0)$.

ii) If $G$ is Abelian there is $H \subset G$ that acts trivially on $\mathrm{M}$, and $G / H$ acts freely on M tangentially positively on $\mu_{G / H}^{-1}(0)=\mu_{G}^{-1}(0)$.

Proof. We first show (0). Given $\zeta$ in $\mathfrak{H}$, the Lie Algebra of $H$, consider the linear endomorphism

$$
\left(\nabla \zeta_{\mathrm{M}}\right)_{p}: T_{p} \mathrm{M} \rightarrow T_{p} \mathrm{M}, \quad z \mapsto \nabla_{z} \zeta_{\mathrm{M}}, \quad \forall z \in T_{p} \mathrm{M} .
$$

Let $\xi \in \mathfrak{G}$ be arbitrary. Since $H \subset G$ is a normal Lie subgroup of $G,[\mathfrak{G}, \mathfrak{H}] \subset \mathfrak{H}$, and thus, since the covariant derivative is torsion-free,

$$
\nabla_{\xi_{\mathrm{M}}(p)} \zeta_{\mathrm{M}}-\nabla_{\zeta_{\mathrm{M}}(p)} \xi_{\mathrm{M}}=\left[\xi_{\mathrm{M}}, \zeta_{\mathrm{M}}\right](p)=[\xi, \zeta]_{\mathrm{M}}(p)=\psi_{\mathrm{M}}(p),
$$

where $\psi \in \mathfrak{H}$. But, if $p \in \operatorname{Fix}(H)$, we have $\zeta_{\mathrm{M}}(p)=\psi_{\mathrm{M}}(p)=0$, which is used in (5.20) to give, since $\xi \in \mathfrak{G}$ was arbitrary,

$$
\mathfrak{G}_{p}^{\mathrm{M}} \subset \operatorname{ker}\left(\nabla \zeta_{\mathrm{M}}\right)_{p} \stackrel{(*)}{=} T_{p}\left(\operatorname{Zero}\left(\zeta_{\mathrm{M}}\right)\right)
$$

where $(*)$ follows easily from the fact used in the proof of Proposition 5.6 that given any geodesic $\gamma$ with $\gamma(0)=p, \zeta_{\mathrm{M}} \circ \gamma$ is a Jacobi field $J$ with initial conditions $J(0)=0$ and $\nabla_{\dot{\gamma}(0)} \zeta_{\mathrm{M}}$. (Alternatively see [10].)

But, in light of (5.21) and the transversality condition (5.12) we must have

$$
\operatorname{ker}\left(\nabla \zeta_{\mathrm{M}}\right)_{p}=T_{p}\left(\operatorname{Zero}\left(\zeta_{\mathrm{M}}\right)\right)=T_{p} \mathrm{M},
$$

and thus, since $\zeta_{\mathrm{M}}(p)=0$ and $\zeta_{\mathrm{M}}$ is a Killing vector field, $\zeta_{\mathrm{M}}$ is identically zero, that is, Zero $\left(\zeta_{\mathrm{M}}\right)=\mathrm{M}$. Since $\zeta$ was arbitrary in $\mathfrak{H}$, the proposition is proved.

We now show the corollaries. For i) simply note that either all the vector fields corresponding to the $H$ action are zero, or no such vector field vanishes at some point in M. For part ii) take $H=\prod_{p_{i} \in \mathrm{M}} \operatorname{Isotr}\left(p_{i}\right)$ and use the fact that any subgroup of $G$, Abelian, is normal.

Corollary 5.7 says that if $G$ is connected and acts nontrivially tangentially positively in $\mu_{G}^{-1}(0)$, it has no fixed points, that is, $\operatorname{dim} \mathfrak{G}_{p}^{\mathrm{M}}>0$ for all $p \in \mathrm{M}$. In the following case we can say a bit more.

Corollary 5.9. Let $G$ connected act on $\mathrm{M}$ tangentially positively on $\mu_{G}^{-1}(0)$. If there is a point $p \in \mathrm{M}$ with $\operatorname{dim} \mathfrak{G}_{p}^{\mathrm{M}}=1$, then $G / \operatorname{Isotr}(p)=\mathbb{R}$ or $\mathbb{S}^{1}$ and $G / \operatorname{Isotr}(p)$ acts freely on $\mathrm{M}$.

Proof. Let $p \in \mathrm{M}$ with $\operatorname{dim} G_{p}^{\mathrm{M}}=1$. If $\operatorname{dim} \operatorname{Isotr}(p)=0$, then $\operatorname{dim} G=1$ and $G=\mathbb{R}$ or $\mathbb{S}^{1}$, which, by Proposition 5.8 , must act freely. So, assume $\operatorname{dim} \operatorname{Isotr}(p)>$ 0 . Let $\zeta$ be in the Lie algebra $\mathfrak{I}_{p} \subset \mathfrak{G}$ of the Lie subgroup $\operatorname{Isotr}(p) \subset G$, and 
consider as before $\left(\nabla \zeta_{\mathrm{M}}\right)_{p}$ as an endomorphism of $T_{p} \mathrm{M}$. Since the action is assumed tangentially positive on $\mu_{G}^{-1}(0)$, condition (5.12) is met at $p$. But since $\left(\nabla \zeta_{\mathrm{M}}\right)_{p}$ is skew-symmetric with respect to $g$ in $T_{p} \mathrm{M}$, as $\zeta_{\mathrm{M}}$ is a Killing vector field, we have the $g$-orthogonal decomposition $T_{p} \mathrm{M}=\operatorname{ker}\left(\nabla \zeta_{\mathrm{M}}\right)_{p} \oplus \operatorname{Image}\left(\left(\nabla \zeta_{\mathrm{M}}\right)_{p}\right)$, and thus (5.12) is equivalent to

$$
\operatorname{Image}\left(\left(\nabla \zeta_{\mathrm{M}}\right)_{p}\right) \subset \mathfrak{G}_{p}^{\mathrm{M}}
$$

Now, by skew-symmetry of $\nabla \zeta_{\mathrm{M}}$ again, $\operatorname{dim} \operatorname{Image}\left(\left(\nabla \zeta_{\mathrm{M}}\right)_{p}\right)$ is even. So since $\operatorname{dim} \mathfrak{G}_{p}=1,\left(\nabla \zeta_{\mathrm{M}}\right)_{p}=0$ and since $\zeta_{\mathrm{M}}(p)=0$, this killing vector field vanishes identically on M. But $\zeta$ was arbitrary in $\mathfrak{I}_{p}$; hence $\operatorname{Isotr}(p)$ fixes every point of G, and thus is a normal subgroup of $G$, with $G / \operatorname{Isotr}(p)$ a one-dimensional connected Lie group, which again, by Proposition 5.8 must act freely on $\mathrm{M}$.

\section{Actions tangentially positive on $\mu_{G}{ }^{-1}(0)$ For Euclidean and for PRoduct Riemannian manifolds. Theorems 6.1, 6.5 and 6.10}

\subsubsection{Euclidean case.}

In [1] we showed that if $G$ is Abelian and acts on Euclidean space tangentially positively on $\mu_{G}^{-1}(0)$, it acts by translations. On the other hand, denoting by $E(s)$ the group of rigid motions of $\mathbb{R}^{s}$, let $\mathrm{R}^{n}$ be acted on by the group $G=\mathrm{E}(k) \subset \mathbb{E}(n)$ that leaves invariant a given $k$-plane, $k \leq n$. This action can be shown directly to be tangentially positive on $\mu_{G}^{-1}(0)$, but it does not consist purely of translations. However, the orbit structure is the same as if it did. We now show that this is a characterizing property.

Theorem 6.1. Let $G$ be a connected Lie group of rigid motions of Euclidean $\mathbb{R}^{n}$. Then $G$ acts tangentially positively in $\mu_{G}^{-1}(0)$ if and only if the orbits are the parallel translates of a given hyperplane. (Moreover, $G$ so acting is Abelian if and only if it consists purely of translations.)

Remark 6.2. In non-flat Riemannian manifolds it is in general not true that the orbits of tangentially positive actions in $\mu_{G}^{-1}(0)$ are totally geodesic submanifolds, as the example of the action (7.6) in hyperbolic space shows.

Proof. First, the statement in parenthesis, included here for completeness, was shown in [1] and is not necessary to prove the rest of the claim.

Fix an origin of $\mathbb{R}^{n}$. The identifications

$$
\mathbb{R}^{n} \cong\left(\begin{array}{c}
\mathbb{R}^{n} \\
1
\end{array}\right) \subset \mathbb{R}^{n+1}, \quad \mathbb{E}(n) \cong\left\{\left(\begin{array}{cc}
\mathbb{O}(n) & \mathbb{R}^{n} \\
0 & 1
\end{array}\right)\right\} \subset \mathbb{G} L(n+1),
$$

exhibits $\mathbb{E}(n)$ as the semi-direct product of the Lie groups of translations of $\mathbb{R}^{n}$ and of rotations $\mathbb{O}(n)$ fixing o. Correspondingly, the Lie algebra of $\mathbb{E}(n)$ splits $\mathfrak{E}(n)=\mathfrak{O}(n)+\mathbb{R}^{n}$.

Let $G \subset \mathbb{E}(n)$. Given $\zeta \in \mathfrak{G}$ we write $\zeta_{\mathbb{R}^{n}}(x)=\zeta_{\mathbb{R}^{n}}^{\text {trans }}(x)+\zeta_{\mathbb{R}^{n}}^{\text {rot }}(x)$ for the action vector field in $\mathbb{R}^{n}$. Explicitly, let $\zeta=\left(\begin{array}{cc}A & u \\ 0 & 0\end{array}\right) \in \mathfrak{E}(n)$, where $A=\left(A_{i j}\right)$ is a skew- 
symmetric $n \times n$ matrix and $u=\left(u_{1}, \ldots, u_{n}\right) \in \mathbb{R}^{n}$. Then, at $x=\left(x_{1}, \cdots, x_{n}\right) \in$ $\mathbb{R}^{n}$,

$$
\zeta_{\mathrm{R}^{n}}(x)=\underbrace{\sum_{i=1}^{n} u_{i} \frac{\partial}{\partial x_{i}}}_{\zeta_{\mathbb{R}^{n}}^{\text {trans }}(x)}+\underbrace{\sum_{i, j=1}^{n} A_{i j} x_{j} \frac{\partial}{\partial x_{i}}}_{\zeta_{\mathbb{R}^{n}}^{\text {rot }}(x)} .
$$

Now, given any hyperplane $\Pi_{\mathbf{o}}$ through o the subgroup $\{\exp (t \zeta), t \in \mathbb{R}\} \subset G$ leaves $\Pi_{\mathbf{o}}$ invariant if and only if $\zeta_{\mathbb{R}^{n}}(x) \in \Pi_{\mathbf{o}}$ for all $x \in \Pi_{\mathbf{o}}$, that is, if and only if (6.1)

$$
\sum_{i=1}^{n} z_{i}\left(u_{i}+\sum_{j=1}^{n} A_{i j} x_{j}\right)=0, \forall z=\left(z_{1}, \ldots, z_{n}\right) \perp \Pi_{\mathbf{o}}, \forall x=\left(x_{1}, \ldots, x_{n}\right) \in \Pi_{\mathbf{o}} .
$$

By considering the case $x=\mathbf{o}$, we can re-write the condition above as

$$
\forall z=\left(z_{1}, \ldots, z_{n}\right) \in \Pi_{\mathbf{o}}\left\{\begin{array}{l}
\sum_{i} z_{i} u_{i}=0, \\
\sum_{i} z_{i} A_{i j}=0 .
\end{array}\right.
$$

But, since the coordinate fields are parallel, and by the skew-symmetry of $A$,

$$
\nabla_{z} \zeta_{\mathbb{R}^{n}}=\sum_{i, j=1}^{n} z_{j} A_{i j} \frac{\partial}{\partial x_{i}}=-\sum_{i, j=1}^{n} z_{j} A_{j i} \frac{\partial}{\partial x_{i}},
$$

in light of which (6.2) becomes

$$
\{\exp (t \zeta), t \in \mathbf{R}\} \cdot \Pi_{\mathbf{o}} \subset \Pi_{\mathbf{o}} \Longleftrightarrow \begin{cases}\forall x \in \Pi_{\mathbf{o}}, & \zeta_{\mathbb{R}^{n}}^{\text {trans }}(x) \in \Pi_{\mathbf{o}} \\ \forall z \perp \Pi_{\mathbf{o}}, & \nabla_{z} \zeta_{\mathbb{R}^{n}}^{r o t}=0 .\end{cases}
$$

On the other hand, since in $T \mathbb{R}^{n}$, it holds that $\mathbf{P}(u)_{z}^{h}=(u)_{z}^{v}$ for all $\zeta \in \mathfrak{G}$, all $u \in T_{x} \mathbb{R}^{n}$, all $x \in \mathbb{R}^{n}$ and all $z \in T_{x} \mathbb{R}^{n}$,

$$
\left\|\zeta_{T \mathbb{R}^{n}}(z)\right\|_{\mathbf{G}_{\mathbf{P}}}^{2}=d \Theta\left(\mathbf{P} \zeta_{T \mathbb{R}^{n}}(z), \zeta_{T \mathbb{R}^{n}}(z)\right)=\left\|\zeta_{\mathbb{R}^{n}}(x)\right\|_{\mathbb{R}^{n}}^{2}-\overbrace{\left\|\nabla_{z} \zeta_{\mathbb{R}^{n}}\right\|_{\mathbb{R}^{n}}^{2}}^{(*)} .
$$

Consequently, the action is tangentially positively in $\mu_{G}^{-1}(0)$ if and only if the righthand side of (6.5) remains non-negative for all $z \in \mu_{G}^{-1}(0)$ and all $\zeta$ in $\mathfrak{G}$, which holds true, due to the presence in (6.5) of the term $(*)$ quadratic in $\|z\|$, if and only if

$$
\nabla_{z} \zeta_{\mathbb{R}^{n}}=0, \forall z \in \mu_{G}^{-1}(0), \forall \zeta \in \mathfrak{G} .
$$

We now prove the necessary condition. Assuming the action tangentially positive in $\mu_{G}^{-1}(0)$ for all $\xi$ and $\zeta$ in $\mathfrak{G}$ and $z \in \mu_{G}^{-1}(0)$,

$$
g\left(\nabla_{\xi_{\mathbb{R}^{n}}} \zeta_{\mathbb{R}^{n}}, z\right)=-g\left(\nabla_{z} \zeta_{\mathbb{R}^{n}}, \xi_{\mathbb{R}^{n}}\right)=0,
$$

from (6.6) and skew-symmetry of $\nabla \zeta_{\mathbb{R}^{n}}$, which shows that the orbits are totallygeodesic, hence hyperplanes. Let $\Pi_{\mathbf{o}}$ be the hyperplane $G \cdot \mathbf{o}$. Then, since for all $\zeta \in \mathfrak{G}, \nabla \zeta_{\mathbb{R}^{n}}^{\text {trans }}=0$, by (6.4),$G$ actually leaves invariant every parallel translate of $\Pi_{\mathbf{o}}=G \cdot \mathbf{o}$. But, by Proposition 5.6, all the orbits have the same dimension, and it follows that each of these parallel translates of $\Pi_{\mathbf{o}}$ is an orbit.

We now prove the sufficient condition. Assume the orbits of a $G$-action are the parallel translates of a hyperplane $\Pi_{\mathbf{o}}=G \cdot \mathbf{o}$. Then $\mu_{G}^{-1}(0)$ consists of all (the parallel translates of) $z \in \mathbb{R}^{n}$ orthogonal to $\Pi_{\mathbf{o}}$. Hence, by (6.4), for all $\zeta \in \mathfrak{G}$, 
$\nabla_{z} \zeta_{\mathbb{R}^{n}}=\nabla_{z} \zeta_{\mathbb{R}^{n}}^{\text {rot }}=0$ for all $z \in \mu_{G}^{-1}(0)$, and, by the argument right after (6.5), the action is tangentially positive on $\mu_{G}^{-1}(0)$.

6.0.8. Riemannian products. We now consider actions on Riemannian products $(\mathrm{M}, g)=\left(\prod_{i=1}^{k} \mathrm{M}_{i}, \prod_{i=1}^{k} g_{i}\right)$. When $(\mathrm{M}, g)$ is (isometric to) such a product, with at least two factors of positive dimension, it is called a reducible Riemannian manifold. In this situation we have the splitting $T \mathrm{M}=\prod_{i=1}^{k} T \mathrm{M}_{i}$; accordingly we express each $z \in T \mathrm{M}$ as $z=\sum_{i=1}^{k} z_{i}$ with $z_{i} \in T \mathrm{M}_{i}$, and in addition,

$$
E(z)=\sum_{i=1}^{k} E_{i}\left(z_{i}\right),\left.\quad \Theta\right|_{T_{z}(T \mathrm{M})}=\left.\sum_{i=1}^{k} \Theta_{i}\right|_{T_{z_{i}}\left(\mathrm{M}_{i}\right)},\left.\quad \mathbf{P}\right|_{T_{z}(T \mathrm{M})}=\left.\sum_{i=1}^{k} \mathbf{P}_{i}\right|_{T_{z_{i}}\left(\mathrm{M}_{i}\right)},
$$

with $\mathbf{P}_{i}$ the adapted structures on $T \mathrm{M}_{i}$. The splitting of $\mathbf{P}$ is a consequence of the fact that each factor $\mathrm{M}_{i}$ is totally geodesic in $\mathrm{M}$. Moreover, the covariant derivative of M splits as

$$
\nabla_{z} w=\sum_{i=1}^{k} \nabla_{z_{i}}^{(i)} w_{i}
$$

and the horizontal and vertical lifts at $z \in T \mathrm{M}$ of $u=\left(\ldots, u_{i}, \ldots\right) \in T \mathrm{M}$, with $u_{i} \in T_{z_{i}} \mathrm{M}_{i}$, split as

$$
(u)_{z}^{h}=\sum_{i=1}^{k}\left(u_{i}\right)_{z_{i}}^{h}, \quad(u)_{z}^{v}=\sum_{i=1}^{k}\left(u_{i}\right)_{z_{i}}^{v} .
$$

Definition 6.3. We say that $G$ acts isometrically on $\mathrm{M}=\prod_{i=1}^{k} \mathrm{M}_{i}$ preserving the splitting if it acts via a group homomorphism

$$
G \rightarrow \prod_{i=1}^{k} \operatorname{Isom}\left(\mathrm{M}_{i}\right) \subset \operatorname{Isom}(\mathrm{M})
$$

Remark 6.4. The degree to which it is restrictive to consider actions satisfying condition (6.10) depends on the splitting of $\mathrm{M}$ considered. For instance, if $\prod_{i} \mathrm{M}_{i}$ is the deRham decomposition of a simply-connected Riemannian manifold (see [11]), any action by a connected $G$ will preserve the splitting.

If an isometric $G$-action preserves a splitting $(\mathrm{M}, g)=\left(\prod_{i=1}^{k} \mathrm{M}_{i}, \prod_{i=1}^{k} g_{i}\right)$, we have by (6.7) and (6.9), for $\xi$ and $\zeta \in \mathfrak{G}$ and $z \in T \mathrm{M}$,

$$
d \Theta\left(\mathbf{P} \xi_{T \mathrm{M}}(z), \zeta_{T \mathrm{M}}(z)\right)=\sum_{i=1}^{k} d \Theta_{i}\left(\mathbf{P}_{i} \xi_{T \mathrm{M}_{i}}\left(z_{i}\right), \zeta_{T \mathrm{M}_{i}}\left(z_{i}\right)\right),
$$

with

$$
\xi_{T \mathrm{M}}(z)=\left(\xi_{\mathrm{M}}(p)\right)_{z}^{h}+\left(\nabla_{z} \xi_{\mathrm{M}}\right)_{z}^{v}=\sum_{i=1}^{k}\left(\xi_{\mathrm{M}_{i}}\left(p_{i}\right)\right)_{z_{i}}^{h}+\left(\nabla_{z_{i}}^{i} \xi_{\mathrm{M}_{i}}\right)_{z_{i}}^{v}
$$

where $\xi_{T \mathrm{M}_{i}}\left(z_{i}\right)=\left(\xi_{\mathrm{M}_{i}}\left(p_{i}\right)\right)_{z_{i}}^{h}+\left(\nabla_{z_{i}}^{i} \xi_{\mathrm{M}_{i}}\right)_{z_{i}}^{v}$ is the action vector field at $z_{i}$ of the tangential $G$-action induced on each factor $T \mathrm{M}_{i}$, and $p=\pi(z)=\left(\ldots, p_{i}, \ldots\right) \in$ 
$\prod \mathrm{M}_{i}$. Thus, by the splitting of $\Theta$ and (6.12), the moment map of the $G$-action on $T \mathrm{M}$ splits as

$$
\mu_{G}(z)=\sum_{i=1}^{k} \mu_{G \mid i}\left(z_{i}\right)
$$

the moment map for the tangential action corresponding to the $G$-action induced on $\mathrm{M}_{i}$ being

$$
\mu_{G \mid i}: T \mathrm{M}_{i} \rightarrow \mathfrak{G}^{*} .
$$

Theorem 6.5. Let $G=\mathbb{R}$ or $\mathbb{S}^{1}$ act non-trivially isometrically on $(\mathrm{M}, g)=$ $\left(\mathrm{M}_{1} \times \mathrm{M}_{2}, g_{1} \times g_{2}\right)$ preserving the splitting where $(\mathrm{M}, g)$ is complete with no conjugate points. Assume that $\mathrm{M}_{1}$ is non-positively curved of rank one, or that it has negative-definite Ricci tensor at some point $p$.

If the action is tangentially positive on $\mu_{G}{ }^{-1}(0)$, then it is trivial in one of the factors and free in the other.

Proof. Consider $G=\mathbb{R}$ or $\mathbb{S}^{1}$. Since the action preserves the splitting, for $\xi=\frac{\partial}{\partial t} \in$ $\mathfrak{G}=\mathbb{R}$ we can define the vector field $X: \mathrm{M}_{1} \times \mathrm{M}_{2} \rightarrow T\left(\mathrm{M}_{1} \times \mathrm{M}_{2}\right)$,

$$
X\left(p_{1}, p_{2}\right)=\left\|\xi_{\mathrm{M}_{2}}\left(p_{2}\right)\right\|_{2}^{2} \xi_{\mathrm{M}_{1}}\left(p_{1}\right)-\left\|\xi_{\mathrm{M}_{1}}\left(p_{1}\right)\right\|_{1}^{2} \xi_{\mathrm{M}_{2}}\left(p_{2}\right),
$$

where $\|u\|_{i}^{2}=g_{i}(u, u)$. By (6.13) the moment map $\mu_{G}: T \mathrm{M} \rightarrow \mathbb{R}$ evaluated at $\xi$ takes the form $\mu_{G}(z)[\xi]=\sum_{i=1}^{2} g_{i}\left(z_{i}, \xi_{\mathrm{M}_{i}}\left(p_{i}\right)\right)$, and so we have $\mu_{G}\left(X\left(p_{1}, p_{2}\right)\right)[\xi]=$ 0 for all $\left(p_{1}, p_{2}\right) \in \mathrm{M}_{1} \times \mathrm{M}_{2}$. Thus, by linearity of $\mu_{G}(z)[\xi]$ in both the $z$ and $\xi$ arguments, and since $\operatorname{dim} G=1$,

$$
\mathcal{S}_{X}=\left\{\mathbb{R} X\left(p_{1}, p_{2}\right) \mid\left(p_{1}, p_{2}\right) \in \mathrm{M}_{1} \times \mathrm{M}_{2}\right\} \subset \mu_{G}^{-1}(0) .
$$

Now,

$$
\begin{aligned}
\nabla_{X\left(p_{1}, p_{2}\right)} X & =\left\|\xi_{\mathrm{M}_{2}}\left(p_{2}\right)\right\|_{2}^{2} \nabla_{\xi_{\mathrm{M}_{1}\left(p_{1}\right)}^{(1)} X-\left\|\xi_{\mathrm{M}_{1}}\left(p_{1}\right)\right\|_{1}^{2} \nabla_{\xi_{\mathrm{M}_{2}\left(p_{2}\right)}^{(2)} X}^{(2)}}=\left\|\xi_{\mathrm{M}_{2}}\left(p_{2}\right)\right\|_{2}^{4} \nabla_{\xi_{\mathrm{M}_{1}}\left(p_{1}\right)}^{(1)} \xi_{\mathrm{M}_{1}}+\left\|\xi_{\mathrm{M}_{1}}\left(p_{1}\right)\right\|_{1}^{4} \nabla_{\xi_{\mathrm{M}_{2}}\left(p_{2}\right)}^{(2)} \xi_{\mathrm{M}_{2}},
\end{aligned}
$$

where we used that:

a) the covariant derivative of $\mathrm{M}$ splits as in (6.8);

b) $\nabla_{\xi_{\mathrm{M}_{i}}\left(p_{i}\right)}^{(i)}\left(\left\|\xi_{\mathrm{M}_{j}}\left(p_{j}\right)\right\|_{j}^{2}\right)=0$ for $i \neq j$ since the variables are independent; and

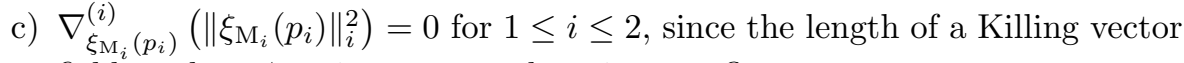
field, such as $\xi_{\mathrm{M}_{i}}$, is constant along its own flow.

But, by hypothesis $G$ acts tangentially positively on $\mu_{G}{ }^{-1}(0)$, and thus, by (6.15), it follows from Theorem 4.1 that $\nabla_{X} X=0$ on $\mathrm{M}_{1} \times \mathrm{M}_{2}$. So, by (6.16)

$$
\forall\left(p_{1}, p_{2}\right) \in \mathrm{M}_{1} \times \mathrm{M}_{2},\left\{\begin{array}{l}
\left\|\xi_{\mathrm{M}_{2}}\left(p_{2}\right)\right\|_{2}^{4} \nabla_{\xi_{\mathrm{M}_{1}}\left(p_{1}\right)}^{(1)} \xi_{\mathrm{M}_{1}}=0 \\
\left\|\xi_{\mathrm{M}_{1}}\left(p_{1}\right)\right\|_{1}^{4} \nabla_{\xi_{\mathrm{M}_{2}}\left(p_{2}\right)}^{(2)} \xi_{\mathrm{M}_{2}}=0 .
\end{array}\right.
$$

It follows that the induced $G$-actions on $\mathrm{M}_{1}$ and $\mathrm{M}_{2}$ cannot both be non-trivial. For, if the action in $\mathrm{M}_{2}$ is non-trivial, there is $p_{2} \in \mathrm{M}_{2}$ with $\xi_{\mathrm{M}_{2}}\left(p_{2}\right) \neq 0$, and by the first equality in (6.17) $\nabla_{\xi_{\mathrm{M}_{1}\left(p_{1}\right)}} \xi_{\mathrm{M}_{1}}=0$ for all $p_{1} \in \mathrm{M}_{1}$. This is impossible by the hypotheses on $\mathrm{M}_{1}$, as shown in the proof of parts 2) and 3) of Corollary [5.4. unless the action on $\mathrm{M}_{1}$ is trivial. 
It remains to show that the action on the one factor, say $\mathrm{M}_{a}$, where $G$ acts non-trivially, is actually free. But, by (6.11), in our situation

$$
\mathbf{G}_{\mathbf{P}}\left(\xi_{T \mathrm{M}}(z), \xi_{T \mathrm{M}}(z)\right)=G_{\mathbf{P}_{a}}\left(\xi_{T \mathrm{M}_{a}}\left(z_{a}\right), \xi_{T \mathrm{M}_{a}}\left(z_{a}\right)\right),
$$

and, since by (6.13) always

$$
\mu_{G}^{-1}(0) \supset\left(\mu_{G \mid 1}^{-1}(0) \times \mu_{G \mid 2}^{-1}(0)\right),
$$

it follows from (6.18) that the action on $\mathrm{M}_{a}$ is tangentially positive in $\mu_{G \mid a}{ }^{-1}(0)$. Such action must be free, by the particular case $\operatorname{dim} G=1$ in Proposition 5.8 applied to $\mathrm{M}_{a}$.

Combining Theorem 1.2 and Theorem 6.5 we have shown the following.

Corollary 6.6. Let $\mathrm{M}=\mathrm{M}_{1} \times \mathrm{M}_{2}$ with no conjugate points with $\mathrm{M}_{1}$ non-positively curved of rank one or with negative-definite Ricci tensor at some point $p_{1}$. Let $\operatorname{dim} G=1$ (resp. $G=\mathbb{R}$ or $\mathbb{S}^{1}$ ) act on $\mathrm{M}$ preserving the splitting with a smooth conjugate-point free quotient $\mathrm{B}=\mathrm{M} / G$. Then $\mathrm{B}$ itself, or one of its factors, is locally isometric (resp. isometric) to $\mathrm{M}_{1}$ or $\mathrm{M}_{2}$. (It could be either factor, but see Remark 6.8.)

Corollary 6.7. Let $(\mathrm{M}, g)$ be a simply connected, complete Riemannian manifold with no conjugate points and let $(\mathrm{M}, g)=\prod_{i}\left(\mathrm{M}_{i}, g_{i}\right)$ be its deRham decomposition. (See [11.) Then any smooth $\mathbb{S}^{1}$-quotient $\mathrm{M} / \mathbb{S}^{1}$ with no conjugate points contains all the non-positively curved factors of rank one present in the deRham decomposition of $\mathrm{M}$.

Proof. Pick a negatively curved factor of rank one in the deRham decomposition $\mathrm{M}=\prod_{i} M_{i}$. By relabeling if necessary, it is $\mathrm{M}_{1}$. The deRham decomposition of $\mathrm{M}$ is unique up to a permutation of isometric factors, and thus $\mathbb{S}^{1}$, being connected, acts on $M$ preserving the splitting $\mathrm{M}=\mathrm{M}_{1} \times \mathrm{M}_{1}^{\prime}$ where $\mathrm{M}_{1}^{\prime}=\prod_{i \neq 1} \mathrm{M}_{i}$. If the $\mathbb{S}^{1}$ action is trivial there is nothing to prove. If it is not, apply Theorem 6.5 to $\mathrm{M}_{1} \times \mathrm{M}_{1}^{\prime}$ and conclude that the action is trivial in $\mathrm{M}_{1}$ and free in $\mathrm{M}_{1}^{\prime}$ or vice versa. But, by a theorem of E. Cartan (Theorem 9.2 in [12]), there are no fixed point-free actions by compact groups on simply connected and non-positively curved complete manifolds. Thus our $\mathbb{S}^{1}$ action is trivial in $\mathrm{M}_{1}$, and this factor appears 'intact' in $\mathrm{M} / \mathbb{S}^{1}$. Eliminate the factor $\mathrm{M}_{1}$ from $\mathrm{M}$ and the corresponding one from $\mathrm{M} / \mathbb{S}^{1}$, and use induction.

Remark 6.8. In light of the result of E. Cartan used in Corollary 6.7, Corollary 6.6 restricted to $G=\mathrm{S}^{1}$ and $\mathrm{M}_{1}$ negatively curved of rank one and, in addition, simply connected (but $\mathrm{M}_{2}$ still arbitrary) implies that B itself, or one of its factors, is isometric to $\mathrm{M}_{1}$.

On the other hand there are free $\mathbb{R}$-actions tangentially positive on $\mu_{G}{ }^{-1}(0)$ for $\mathrm{H}^{n}$. See examples in Section 7 .

Proposition 6.9. Let $(\mathrm{M}, g)=\left(\mathrm{M}_{1} \times \mathrm{M}_{2}, g_{1} \times g_{2}\right)$ be a complete Riemannian product manifold without conjugate points. Let $G$ act isometrically on $\mathrm{M}$ preserving the splitting and tangentially positive on $\mu_{G}{ }^{-1}(0)$. If for a given $\xi \in \mathfrak{G}$ there is a map $\sigma^{\xi}: \mathrm{M}_{1} \rightarrow \Gamma\left(\mathrm{M}_{2}, T \mathrm{M}_{2}\right)$ (= smooth sections of the tangent bundle) such that

$$
\mu_{G \mid 2}\left(\sigma^{\xi}\left(p_{1}\right)\left[p_{2}\right]\right)=\mu_{G \mid 1}\left(\xi_{\mathrm{M}_{1}}\left(p_{1}\right)\right) \in \mathfrak{G}^{*},
$$

with $\mu_{G \mid i}$ as in (6.14), then $\xi_{\mathrm{M}_{1}}$ is self-parallel. 
Proof. Regarding both $\sigma^{\xi}$ and $\xi_{\mathrm{M}_{1}}$ as vector fields in M by the rules $\left(p_{1}, p_{2}\right) \mapsto$ $\sigma^{\xi}\left(p_{1}\right)\left[p_{2}\right] \in T_{\left(p_{1}, p_{2}\right)} \mathrm{M}$ and $\left(p_{1}, p_{2}\right) \mapsto \xi_{\mathrm{M}_{1}}\left(p_{1}\right) \in T_{\left(p_{1}, p_{2}\right)} \mathrm{M}$, condition (6.20) implies that the vector field

$$
Y^{\xi}=\xi_{\mathrm{M}_{1}}-\sigma^{\xi}: \mathrm{M} \rightarrow T \mathrm{M}
$$

satisfies

$$
\mathcal{S}_{Y^{\xi}}=\left\{\mathbb{R} Y^{\xi}\left(p_{1}, p_{2}\right) \mid\left(p_{1}, p_{2}\right) \in \mathrm{M}\right\} \subset \mu_{G}^{-1}(0),
$$

since for all $\zeta \in \mathfrak{G}$

$$
\begin{aligned}
\mu_{G}\left(Y^{\xi}\left(p_{1}, p_{2}\right)\right)[\zeta] & =g\left(Y^{\xi}\left(p_{1}, p_{2}\right), \zeta_{\mathrm{M}}\left(p_{1}, p_{2}\right)\right) \\
& =g_{1}\left(\xi_{\mathrm{M}_{1}}\left(p_{1}\right), \zeta_{\mathrm{M}_{1}}\left(p_{1}\right)\right)-g_{2}\left(\sigma^{\xi}\left(p_{1}\right)\left[p_{2}\right], \zeta_{\mathrm{M}_{2}}\left(p_{2}\right)\right) \\
& =\mu_{G \mid 1}\left(\xi_{\mathrm{M}_{1}}\left(p_{1}\right)\right)[\zeta]-\mu_{G \mid 2}\left(\sigma^{\xi}\left(p_{1}\right)\left[p_{2}\right]\right)[\zeta] \\
& =0 .
\end{aligned}
$$

Thus, by Theorem 4.1, since by hypothesis the action is tangentially positive on $\mu_{G}^{-1}(0)$ we have $\nabla_{Y^{\xi}} Y^{\xi}=0$.

Let $p_{i}: \mathrm{M} \rightarrow \mathrm{M}_{i}$ be the projections onto the factors of the product. Since $\left(p_{1}\right)_{*} \sigma^{\xi}=0$, it follows from the splitting of the covariant derivative (6.8) that $\nabla_{\sigma^{\xi}} \xi_{\mathrm{M}_{1}}=0$, and hence

$$
\nabla_{Y^{\xi}} Y^{\xi}=\underbrace{\nabla_{\xi_{\mathrm{M}_{1}}} \xi_{\mathrm{M}_{1}}}_{\in \operatorname{ker}\left(p_{2}\right)_{*}}-\underbrace{\nabla_{Y^{\xi}} \sigma^{\xi}}_{\in \operatorname{ker}\left(p_{1}\right)_{*}} .
$$

It follows in particular that $\nabla_{\xi_{\mathrm{M}_{1}}} \xi_{\mathrm{M}_{1}}=\nabla_{\xi_{\mathrm{M}_{1}}}^{(1)} \xi_{\mathrm{M}_{1}}=0$.

Theorem 6.10. Let $\left(\mathrm{M}=\mathrm{M}_{1} \times \mathrm{M}_{2}, g_{1} \times g_{2}\right)$ be a complete Riemannian product with no conjugate points. Let $G$ connected act on $\mathrm{M}$ isometrically preserving the splitting and act freely on $\mathrm{M}_{2}$ so that the quotient $\mathrm{B}=\mathrm{M}_{1} \times{ }_{G} \mathrm{M}_{2}$ with the submersion metric has no conjugate points. Then,

i) if $\mathrm{M}_{1}$ have negative-definite Ricci tensor at some point $p$, the action on $\mathrm{M}_{1}$ is trivial; or

ii) if $\mathrm{M}_{1}$ is non-positively curved of rank $k$, the homomorphism $G \rightarrow \operatorname{Isom}\left(\mathrm{M}_{1}\right)$ defined by the induced action on $\mathrm{M}_{1}$ has kernel of co-dimension $k-1$.

Proof. We pick $\left\{\psi^{1}, \ldots, \psi^{\operatorname{dim} G}\right\}$, a basis for $\mathfrak{G}$. Given $\xi \in \mathfrak{G}$ we define functions

$$
\alpha_{i}^{\xi}: \mathrm{M}_{1} \times \mathrm{M}_{2} \rightarrow \mathbb{R}, \quad 1 \leq i \leq \operatorname{dim} G
$$

so that for all $1 \leq j \leq \operatorname{dim} G$,

$$
\sum_{i=1}^{\operatorname{dim} G} \alpha_{i}^{\xi}\left(p_{1}, p_{2}\right) g_{2}\left(\psi_{\mathrm{M}_{2}}^{i}\left(p_{2}\right), \psi_{\mathrm{M}_{2}}^{j}\left(p_{2}\right)\right)=g_{1}\left(\xi_{\mathrm{M}_{1}}\left(p_{1}\right), \psi_{\mathrm{M}_{1}}^{j}\left(p_{1}\right)\right) .
$$

The functions $\alpha_{i}^{\xi}$ are well defined since the action induced on $\mathrm{M}_{2}$ is assumed free, which implies that the $\operatorname{dim} G \times \operatorname{dim} G$ matrix with $i, j$ entry $g_{2}\left(\psi_{\mathrm{M}_{2}}^{i}\left(p_{2}\right), \psi_{\mathrm{M}_{2}}^{j}\left(p_{2}\right)\right)$ is non-singular.

We define $\sigma^{\xi}: \mathrm{M}_{1} \rightarrow \Gamma\left(\mathrm{M}_{2}, T \mathrm{M}_{2}\right)$ by

$$
\sigma^{\xi}\left(p_{1}\right)\left[p_{2}\right]=\sum_{i=1}^{n} \alpha_{i}\left(p_{1}, p_{2}\right) \psi_{\mathrm{M}_{2}}^{i}\left(p_{2}\right) .
$$


Now, let an element $\psi^{j}$ in the basis of $\mathfrak{G}$ be given. For all $\left(p_{1}, p_{2}\right) \in \mathrm{M}_{1} \times \mathrm{M}_{2}$ and all $\xi \in \mathfrak{G}$, recalling (6.14),

$$
\begin{aligned}
\mu_{G \mid 2}\left(\sigma^{\xi}\left(p_{1}\right)\left[p_{2}\right]\right)\left[\psi^{j}\right] & =g_{2}\left(\sigma^{\xi}\left(p_{1}\right)\left[p_{2}\right], \psi_{\mathrm{M}_{2}}^{j}\left(p_{2}\right)\right) \\
& =\sum_{i=1}^{\operatorname{dim} G} \alpha_{i}^{\xi}\left(p_{1}, p_{2}\right) g_{2}\left(\psi_{\mathrm{M}_{2}}^{i}\left(p_{2}\right), \psi_{\mathrm{M}_{2}}^{j}\left(p_{2}\right)\right) \\
& =g_{1}\left(\xi_{\mathrm{M}_{1}}\left(p_{1}\right), \psi_{\mathrm{M}_{1}}^{j}\left(p_{1}\right)\right) \\
& =\mu_{G \mid 1}\left(\xi_{\mathrm{M}_{1}}\left(p_{1}\right)\right)\left[\psi^{j}\right] .
\end{aligned}
$$

Hence $\sigma^{\xi}$ satisfies (6.20) for all $\xi$. It follows that the action vector fields $\xi_{\mathrm{M}_{1}}$ are self-parallel. Now, to show i) and ii) use the argument in the proof of Corollary 5.4 .

Corollary 6.11. Let $\mathrm{B}=\mathrm{M}_{1} \times{ }_{G} \mathrm{M}_{2}$ as in Theorem 6.10 with $\mathrm{M}_{1}$ with negativedefinite Ricci tensor at some point $p$ or non-positively curved of rank $k=1$. Then, $\mathrm{B}$ is isometric to or has a factor isometric to $\mathrm{M}_{1}$.

Corollary 6.12. Let $\left(\mathrm{M}_{1}, g_{1}\right)$ be complete and with no conjugate points, and have a negative-definite Ricci tensor be at some point $p$ or be non-positively curved of rank one. If $G$, connected, acts diagonally on $\mathrm{M}=\mathrm{M}_{1} \times \mathrm{M}_{1}$ freely and properly with conjugate-point free quotient, then $G=\{1\}$.

Corollary 6.11 says in particular that we cannot deform the metric $g_{1}$ in $\mathrm{M}_{1}$ with the stated properties into a new Riemannian metric with no conjugate points by the usual method $\mathrm{M}_{1} \times_{G} G$, where $G$ is a non-positively curved Lie group acting on itself by group multiplication and non-trivially on $\left(\mathrm{M}_{1}, g_{1}\right)$.

Corollary 6.13. Let $G$ compact and connected act freely on the complete (M, $g$ ) which is assumed with no conjugate points, and with smooth quotient $\left(\mathrm{B}, g_{\mathrm{B}}\right)$ also with no conjugate points. Let $(\mathrm{M}, g)=\prod_{i}\left(\mathrm{M}_{i}, g_{i}\right)$, where each $\left(\mathrm{M}_{i}, g_{i}\right)$ is irreducible or a Euclidean flat space. Then the decomposition of $\mathrm{B}$ into irreducible or Euclidean factors contains every simply connected non-positively curved factor in the decomposition of $\mathrm{M}$.

Proof. Write $\mathrm{M}=\mathrm{M}_{1} \times \mathrm{M}_{2}$, where $\mathrm{M}_{1}$ is the product of all the simply connected non-positively curved factors in the decomposition of M. Clearly $G$ preserves this decomposition. By the Theorem of E. Cartan used in Corollary 6.7 the $G$-action in $\mathrm{M}_{1}$ has a fixed point $p_{1} \in \mathrm{M}_{1}$, and thus, since the action in $\mathrm{M}$ is free by hypothesis, it must be free on $\mathrm{M}_{2}$. By the reasoning in Theorem 6.10 and Proposition 6.9, every action vector field in $\mathrm{M}_{1}$ is self-parallel, $\nabla_{\xi_{\mathrm{M}_{1}}} \xi_{\mathrm{M}_{1}}=0$, and hence of constant length as shown in the proof of Proposition 5.1. But they are all zero at the fixed point $p_{1}$, so the action on $\mathrm{M}_{1}$ is trivial.

\section{ApPENDIX}

We shall illustrate some of the points made above with examples involving hyperbolic space $\mathrm{H}^{n}$. First, we obtain the expression (7.4) for the $\mathbf{G}_{\mathbf{P}}$-length of a tangential action vector field and use it to illustrate the necessary condition in Theorem 4.1. We then show explicitly that the action (7.6) is tangentially positive in $\mu_{G}{ }^{-1}(0)$, but not so in all of $T \mathrm{H}^{n}$. Finally we show explicitly that the action 
(7.8) on the product $\mathrm{H}^{n} \times \mathbb{R}$ fails to be tangentially positive in $\mu_{G}{ }^{-1}(0)$, as predicted by Theorem 6.5

Let $\mathrm{H}^{n}=\left\{\left(x_{1}, \ldots, x_{n}\right) \mid x_{i} \in \mathbb{R}, x_{n}>0\right\}$ with the hyperbolic metric of constant sectional curvature $-1, g=x_{n}^{-2} \sum_{i=1}^{n} d x_{i}^{2}$. Each $z=\left.\sum_{i=1}^{n} u_{i} \frac{\partial}{\partial x_{i}}\right|_{\left(x_{1}, \ldots, x_{n}\right)} \in T \mathrm{H}^{n}$ is identified with the Real $2 n$-tuple $\left(x_{1}, \ldots, x_{n}, u_{1}, \ldots, u_{n}\right)$, and thus we have $T \mathrm{H}^{n}=$ $\left\{\left(x_{1}, \ldots, x_{n}, u_{1}, \ldots, u_{n}\right) \mid x_{i}, u_{i} \in \mathbb{R}, 0<x_{n}\right\}$.

Let $G$ act isometrically on $\mathrm{H}^{n}$. The moment map of the tangential action $\mu_{G}: T \mathrm{H}^{n} \rightarrow \mathfrak{G}^{*}$ satisfies $\mu_{G}(z)[\xi]=\Theta\left(\xi_{T \mathrm{H}^{n}}(z)\right)=g\left(z, \xi_{\mathrm{H}^{n}}(p)\right)$ for all $z \in T_{p} \mathrm{H}^{n}$ and all $\xi \in \mathfrak{G}$.

We need to calculate $\mathbf{P} \xi_{T \mathrm{H}^{n}}(z)$ in terms of $\xi_{\mathrm{H}}^{n}$ and its covariant derivative, and, in light of (2.8), we assume $0 \neq z \in T H^{n}$. From (4.22) for instance, with $\|z\|^{2}=$ $x_{n}^{-2} \sum_{i=1}^{n} u_{i}^{2}$,

$$
\mathbf{P}(w)_{z}^{h}=f(\|z\|)(w)_{z}^{v} \text { if } g(w, z)=0, \quad \mathbf{P}(z)_{z}^{h}=(z)_{z}^{v},
$$

where $f(x)=x \operatorname{coth} x$.

Given $z \in T_{p} \mathrm{H}^{n}$, since $\xi_{\mathrm{H}^{n}}$ is a Killing vector field and thus $\nabla_{\xi_{\mathrm{H}^{n}}}$ is skewsymmetric, $g\left(\nabla_{z} \xi_{\mathrm{H}^{n}}, z\right)=0$, hence using (7.1),$\xi_{T \mathrm{H}^{n}}(z)=\left(\xi_{\mathrm{H}^{n}}(p)\right)_{z}^{h}+\left(\nabla_{z} \xi_{\mathrm{H}^{n}}\right)_{z}^{v}$, and writing

$$
\xi_{\mathrm{H}^{n}}(p)=\left(\xi_{\mathrm{H}^{n}}(p)-\|z\|^{-2} \mu_{G}(z)[\xi] z\right)+\|z\|^{-2} \mu_{G}(z)[\xi] z,
$$

with the expression in (7.2) in parenthesis orthogonal to $z$, it follows that

$$
\begin{aligned}
\mathbf{P} \xi_{T \mathrm{H}^{n}}(z)= & \mu_{G}(z)[\xi]\|z\|^{-2}(1-f(\|z\|))(z)_{z}^{v} \\
& +f(\|z\|)\left(\xi_{\mathrm{H}^{n}}(p)\right)_{z}^{v}+\frac{1}{f(\|z\|)}\left(\nabla_{z} \xi_{\mathrm{H}^{n}}\right)_{z}^{h} .
\end{aligned}
$$

Thus, using (2.4), for all $z \in T_{p} \mathrm{H}^{n}$,

$$
\begin{gathered}
\mathbf{G}_{\mathbf{P}}\left(\xi_{T \mathrm{H}^{n}}(z), \xi_{T \mathrm{H}^{n}}(z)\right)=f(\|z\|)\left\|\xi_{\mathrm{H}^{n}}(p)\right\|^{2}-\frac{1}{f(\|z\|)}\left\|\nabla_{z} \xi_{\mathrm{H}^{n}}\right\|^{2} \\
+\frac{\left(\mu_{G}(z)[\xi]\right)^{2}}{\|z\|^{2}}(1-f(\|z\|)) .
\end{gathered}
$$

Re-arranging, putting whenever $\left\|\xi_{\mathrm{H}^{n}}(p)\right\| \neq 0, \cos \zeta=\|z\|^{-1}\left\|\xi_{\mathrm{H}^{n}}(p)\right\|^{-1} \mu_{G}(z)[\xi]$ (where $\zeta=\zeta(z, \xi) \in \mathbb{R}$ by Schwartz inequality), and evaluating at $a z \in T \mathrm{H}^{n}$ where $0<a \in \mathbb{R}$,

$$
\begin{gathered}
\frac{\mathbf{G}_{\mathbf{P}}\left(\xi_{T \mathrm{H}^{n}}(a z), \xi_{T \mathrm{H}^{n}}(a z)\right)}{\left\|\xi_{\mathrm{H}^{n}}(p)\right\|^{2}} \\
=a\left(\|z\| \operatorname{coth}(\|a z\|) \sin ^{2} \zeta-\|z\|^{-1} \tanh (\|a z\|) \frac{\left\|\nabla_{z} \xi_{\mathrm{H}^{n}}\right\|^{2}}{\left\|\xi_{\mathrm{H}^{n}}(p)\right\|^{2}}\right)+\cos ^{2} \zeta .
\end{gathered}
$$

The formula above allows us to easily note the following: For $a<<1$ the value of (7.5) is positive; we actually see that its limit as $a \mapsto 0$ is 1 , as it should, since $\mathbf{G}_{\mathbf{P}}$ restricts to $g$ along the zero section. This shows explicitly that if the action is free, it is tangentially positively in a neighborhood of the zero section, as indicated by Proposition 3.6. On the other hand if $z$ is such that $\sin \zeta<<1$, by taking $a>>1$ (7.5) can be made negative, unless $\nabla_{z} \xi_{\mathrm{H}^{n}}=0$. This illustrates that, in general, the action cannot be tangentially positive on $\mathfrak{G}^{\mathrm{H}^{n}}$ unless the action vector fields on $\mathrm{M}$ are self-parallel, as shown in Theorem 4.1 . 
However the action may still be tangentially positive on $\mu_{G}{ }^{-1}(0) \subset T \mathrm{H}^{n}$ even if the action vector fields are not parallel. As an example consider the isometric $R$-action

$$
t \cdot\left(x_{1}, \ldots, x_{n}\right)=\left(e^{t} x_{1}, \ldots, e^{t} x_{n}\right) .
$$

Let $\xi=\frac{\partial}{\partial t} \in \mathbb{R}$ be a basis for the Lie algebra of $G, \mathfrak{G}=\mathbb{R}$. The corresponding action vector field on $\mathrm{H}^{n}$ and on $T \mathrm{H}^{n}$ for the tangential action are $\xi_{\mathrm{H}^{n}}\left(x_{1}, \ldots, x_{n}\right)=$ $\sum_{i=1}^{n} x_{i} \frac{\partial}{\partial x_{i}}$ and $\xi_{T \mathrm{H}^{n}}(z)=\sum_{i=1}^{n}\left(x_{i} \frac{\partial}{\partial x_{i}}+u_{i} \frac{\partial}{\partial u_{i}}\right)$. The moment map is $\mu_{G}(z)[\xi]=$ $x_{n}^{-2} \sum_{i=1}^{n} x_{i} u_{i}$. For $1 \leq i, j, k \leq n$, with $\delta_{i j}$ the Kroenecker delta:

$$
\nabla_{\frac{\partial}{\partial x_{i}}} \frac{\partial}{\partial x_{j}}=\frac{\delta_{i j}}{x_{n}} \frac{\partial}{\partial x_{n}}, i \neq n \neq j, \quad \nabla_{\frac{\partial}{\partial x_{i}}} \frac{\partial}{\partial x_{n}}=\nabla_{\frac{\partial}{\partial x_{n}}} \frac{\partial}{\partial x_{i}}=-\frac{1}{x_{n}} \frac{\partial}{\partial x_{i}}, 1 \leq i \leq n .
$$

We calculate at $z=\sum_{i=1}^{n} u_{i} \frac{\partial}{\partial x_{i}} \in T_{p} \mathrm{H}^{n}$ with $p=\left(x_{1}, \ldots, x_{n}\right)$

$$
\nabla_{z} \xi_{\mathrm{H}^{n}}=-\frac{u_{n}}{x_{n}} \sum_{i=1}^{n} x_{i} \frac{\partial}{\partial x_{i}}+\left(\sum_{i=1}^{n} \frac{u_{i} x_{i}}{x_{n}^{2}}\right) x_{n} \frac{\partial}{\partial x_{n}}=-\frac{u_{n}}{x_{n}} \xi_{\mathrm{H}^{n}}(p)+\mu_{G}(z)[\xi] x_{n} \frac{\partial}{\partial x_{n}} .
$$

It follows that

$$
\mathbf{G}_{\mathbf{P}}\left(\xi_{T \mathrm{H}^{n}}(z), \xi_{T \mathrm{H}^{n}}(z)\right)=\left\|\xi_{\mathrm{H}^{n}}(p)\right\|^{2}\left(f(\|z\|)-\frac{u_{n}^{2}}{x_{n}^{2}} \frac{1}{f(\|z\|)}\right)+h(z) \mu_{G}(z)[\xi],
$$

for a certain continuous function $h: T H^{n} \rightarrow \mathbb{R}$ that we do not need to write explicitly. Thus if $\mu_{G}(z)[\xi]=0$ we see that $\mathbf{G}_{\mathbf{P}}\left(\xi_{T \mathrm{H}^{n}}(z), \xi_{T \mathrm{H}^{n}}(z)\right)>0$ since $u_{n}^{2} / x_{n}^{2} \leq\|z\|^{2}$ and $\tanh ^{2}\|z\|<1$. This shows that the $\mathbb{R}$-action (17.6) is tangentially positive on $\mu_{G}{ }^{-1}(0)$. Incidentally, as proved in $\left[1\right.$, the quotient $\mathrm{H}^{n} / \mathbb{R}$ is isometric to $\mathrm{H}^{n-1}$.

Let us check directly that the $\mathbb{R}$-action (7.6) is not tangentially positive on $\mathfrak{G}^{\mathrm{H}^{n}}$. Take $z=\xi_{\mathrm{H}^{n}}(p)$ in (7.5); then $\mu_{G}(z)[\xi]=\left\|\xi_{\mathrm{H}^{n}}(p)\right\|^{2}=x_{n}^{-2} \sum_{i=1}^{n} x_{i}^{2} \geq 1, \cos \zeta=1$, and

$$
\left\|\nabla_{z} \xi_{\mathrm{H}^{n}}\right\|^{2}=\left\|\nabla_{\xi_{\mathrm{H}^{n}}(p)} \xi_{\mathrm{H}^{n}}\right\|^{2}=\left\|\xi_{\mathrm{H}^{n}}(p)\right\|^{2}\left(\left\|\xi_{\mathrm{H}^{n}}(p)\right\|^{2}-1\right) .
$$

So (7.5) becomes

$$
\begin{aligned}
& \left.\frac{\mathbf{G}_{\mathbf{P}}\left(\xi_{T \mathrm{H}^{n}}(a z), \xi_{T \mathrm{H}^{n}}(a z)\right)}{\left\|\xi_{\mathrm{H}^{n}}(p)\right\|^{2}}\right|_{z=\xi_{\mathrm{H}^{n}}(p)} \\
= & 1-a\left\|\xi_{\mathrm{H}^{n}}(p)\right\|^{-1} \tanh \left(a\left\|\xi_{\left.\mathrm{H}^{(} p\right)}\right\|\right)\left(\left\|\xi_{\mathrm{H}^{n}}(p)\right\|^{2}-1\right),
\end{aligned}
$$

where we assumed $0<a \in \mathbb{R}$. But for $p$ not in $\left\{0=x_{1}=\cdots=x_{n-1}\right\} \subset \mathrm{H}^{n}$, where $\left\|\xi_{\mathrm{H}^{n}}(p)\right\|=1$, the expression above can be made negative by taking $a>>1$.

7.0.9. Finally, take the $\mathbb{R}$-action on the Riemannian product $\mathrm{M}=\mathrm{H}^{n} \times \mathbb{R}$ with $\mathbb{R}$ Euclidean,

$$
t \cdot(x, r)=(t \cdot x, r+t),
$$

where $t \cdot x$ is the action on $\mathrm{H}^{n}$ given by (7.6). Since the action is non-trivial in both factors of the Riemannian product, by Theorem 6.5, applied to $\mathrm{M}_{1}=\mathrm{H}^{n}$, the action (7.8) cannot be tangentially positive on $\mu_{G}{ }^{-1}(0)$, which we will now verify directly. Note that in light of Theorem 1.2, this will prove that the quotient $H^{n} \times \mathbb{R}$ has conjugate points. 
It follows from (6.7), (6.11) and (6.12) that for $\zeta=\frac{\partial}{\partial t} \in \mathfrak{G}=\mathbb{R}$,

$$
\begin{aligned}
\mathbf{G}_{\mathbf{P}}\left(\zeta_{T \mathrm{M}}(z), \zeta_{T \mathrm{M}}(z)\right) & =G_{\mathbf{P}_{1}}\left(\zeta_{T \mathrm{H}^{n}}\left(z_{1}\right), \zeta_{T \mathrm{H}^{n}}\left(z_{1}\right)\right)+G_{\mathbf{P}_{2}}\left(\zeta_{T \mathbb{R}}\left(z_{2}\right), \zeta_{T \mathbb{R}}\left(z_{2}\right)\right) \\
& =G_{\mathbf{P}_{1}}\left(\zeta_{T \mathrm{H}^{n}}\left(z_{1}\right), \zeta_{T \mathrm{H}^{n}}\left(z_{1}\right)\right)+1
\end{aligned}
$$

where we used that $G_{\mathbf{P}_{2}}\left(\zeta_{T \mathbb{R}}\left(z_{2}\right), \zeta_{T \mathbb{R}}\left(z_{2}\right)\right)=\left\|\zeta_{\mathbb{R}}\left(p_{2}\right)\right\|_{\mathbb{R}}^{2}=1$, true by (4.22) and the fact that $\zeta_{\mathbb{R}}$ is parallel of length 1 in $\mathbb{R}$ Euclidean. ( $\mathbf{P}_{2}$ is just the structure $\mathbf{P}_{0}$ with product coordinates as in (2.9) .)

The expression (7.9), valid at all $z \in T \mathrm{M}$, if evaluated at

$$
z=\underbrace{-a \zeta_{\mathrm{H}^{n}}\left(p_{1}\right)}_{=z_{1} \in T \mathrm{H}^{n}}+\underbrace{a\left\|\zeta_{\mathrm{H}^{n}}\left(p_{1}\right)\right\|_{\mathrm{H}^{n}}^{2} \zeta_{\mathbb{R}}}_{=z_{2} \in T \mathbb{R}} \in \mu_{G}^{-1}(0) \subset T \mathrm{M},
$$

can be made negative by taking $a>>1$ by the calculation (7.7), provided that we pick $p_{1}$ not on the $x_{n}$ axis in $\mathrm{H}^{n}$. So, the action is not tangentially positive on $\mu_{G}^{-1}(0)$.

7.0.10. A related complex structure. Our motivation for defining $\mathbf{P}$ in terms of the symplectic geometry of TM comes from the construction of the so-called adapted complex structure $\mathbf{J}$ in TM by L. Lempert and R. Szöke in 13 and independently by V. Guillemin and M. Stenzel in $T^{*} \mathrm{M}$ in [6], defined to characterize certain Stein manifolds, the Grauert tubes. We view conjugate point-free Riemannian manifolds as analogs of those for which $\mathbf{J}$ is defined on the whole $T \mathrm{M}$ which hence becomes an unbounded Grauert tube, i.e., with unbounded Monge-Ampère exhaustion; the naturality of $\mathbf{J}$ under the Kähler reduction (8]) we have shown in 2] motivates the approach on conjugate points in the context of isometric actions via a PseudoRiemannian reduction involving $\mathbf{P}$. In the Kähler reduction case the $\mathbf{J}$-invariant connection on $\mu_{G}^{-1}(0)$ is given by a positive-definite metric, and thus is defined as long as $\mathbf{J}$ itself is, so the naturality of the reduction allows us to produce new examples of unbounded Grauert tubes by isometric quotients. On the other hand, the eventual failure of the pseudo-Riemannian reduction away from the zero section corresponds to the creation of conjugate points, and naturality of the reduction can then be used for non-existence results.

We end with the simplest illustration of the reduction of $\mathbf{J}$ versus that of $\mathbf{P}$ in the same space. Let $\mathrm{M}=\mathbb{R}^{2} \times \mathbb{R}$ with the Euclidean metric, and let $T \mathbb{R}^{3} \cong \mathbf{R}^{6}$ where $(x, y, r, u, v, t))$ are the coordinates of $z=u \frac{\partial}{\partial x}+v \frac{\partial}{\partial y}+t \frac{\partial}{\partial r} \in T \mathbb{R}^{3}$. Here $d \Theta=d u \wedge d x+d v \wedge d y+d t \wedge d r$. Let $G=\mathbb{R}$ act freely isometrically on $\mathbb{R}^{3}$ by $(x, y, r) \stackrel{\theta}{\mapsto}(x \cos \theta-y \sin \theta, x \sin \theta+y \cos \theta, r+\theta)$, with $\xi_{\mathbb{R}^{3}}(x, y, r)=-y \frac{\partial}{\partial x}+x \frac{\partial}{\partial y}+$ $\frac{\partial}{\partial r}$, and $\xi_{T \mathbb{R}^{3}}(z)=-y \frac{\partial}{\partial x}+x \frac{\partial}{\partial y}+\frac{\partial}{\partial r}-v \frac{\partial}{\partial u}+u \frac{\partial}{\partial v}$.

In the Euclidean case both structures $\mathbf{P}$ and $\mathbf{J}$ are defined in the entire TM. Explicitly, $\mathbf{P} \frac{\partial}{\partial x}=\frac{\partial}{\partial u}, \mathbf{P} \frac{\partial}{\partial y}=\frac{\partial}{\partial v}, \mathbf{P} \frac{\partial}{\partial r}=\frac{\partial}{\partial t}$ and $\mathbf{P}^{2}=\mathbf{I}$, while $\mathbf{J} \frac{\partial}{\partial x}=\frac{\partial}{\partial u}$, $\mathbf{J} \frac{\partial}{\partial y}=\frac{\partial}{\partial v}, \mathbf{J} \frac{\partial}{\partial r}=\frac{\partial}{\partial t}$ and $\mathbf{J}^{2}=-\mathbf{I}$. So, $\mathbf{P} \xi_{T \mathbb{R}^{3}}(z)=-y \frac{\partial}{\partial u}+x \frac{\partial}{\partial v}+\frac{\partial}{\partial t}-v \frac{\partial}{\partial x}+u \frac{\partial}{\partial y}$ and $\mathbf{J} \xi_{T \mathbb{R}^{3}}(z)=-y \frac{\partial}{\partial u}+x \frac{\partial}{\partial v}+\frac{\partial}{\partial t}+v \frac{\partial}{\partial x}-u \frac{\partial}{\partial y}$. Now, both $\xi_{T \mathbb{R}^{3}} \wedge \mathbf{P} \xi_{T \mathbb{R}^{3}} \neq 0$ and $\xi_{T \mathbb{R}^{3}} \wedge \mathbf{J} \xi_{T \mathbb{R}^{3}} \neq 0$ on $T \mathbb{R}^{3}$ (as predicted by Proposition 3.4). But since

$$
\begin{gathered}
d \Theta\left(\mathbf{P} \xi_{T \mathbb{R}^{3}}(z), \xi_{T \mathbb{R}^{3}}(z)\right)=1+x^{2}+y^{2}-v^{2}-u^{2}, \\
d \Theta\left(\mathbf{J} \xi_{T \mathbb{R}^{3}}(z), \xi_{T \mathbb{R}^{3}}(z)\right) \stackrel{(*)}{=} 1+x^{2}+y^{2}+v^{2}+u^{2},
\end{gathered}
$$

the $\mathbf{G}_{\mathbf{P}}$-length of the action vector field is negative enough away from the zero section, unlike its Kähler-metric length $(*)$; at points in $\mu_{G}{ }^{-1}(0)$ far away from 
$\mathrm{M}$ the $\mathbf{G}_{\mathbf{P}}$-orthogonal complement to the orbits of the action in $\mu_{G}^{-1}(0)$ intersects the orbits non-transversally, and the connection given by $\mathbf{G}_{\mathbf{P}}$ breaks down. (This is predicted by Theorem 6.1 since the orbits of points other than the origin are helices. See also [1.)

\section{REFERENCES}

[1] Aguilar, R. Moment map, a product structure and Riemannian metrics with no conjugate points. Comm. Anal. Geom. 13 (2005), 401-438. MR2154825

[2] Symplectic reduction and the complex homogeneous Monge-Ampère equation. Ann. Glob. Analysis \& Geom. 19 (2001), 327-353. MR.1842574 (2002g:53144)

[3] Bochner, S. Vector fields and Ricci curvature. Bull. Amer. Math. Soc. 52 (1946), 776-797. MR0018022 (8:230a)

[4] Bourbaki, N. Lie groups and Lie algebra. Chapters 1-3, Springer-Verlag, Berlin (1989). MR0979493 (89k:17001)

[5] Dombrowski., P. On the geometry of the tangent bundle. J. Reine Angew. Math. 210 (1961), 73-88. MR0141050 (25:4463)

[6] Guillemin, V. \& Stenzel, M. Grauert tubes and the homogeneous Monge-Ampère equation I. J. Diff. Geometry 34 (1991), 561-570. MR1131444 (93e:32018)

[7] Hermann, R. A sufficient condition that a mapping of Riemannian manifolds be a fiber bundle. Proc. A.M.S. 11 (1960), 239-242. MR0112151(22:3006)

[8] Hitchin, N., Karlhede, A., Linstrtröm, U., Roček, M. Hyper-Kähler metrics and supersymmetry. Comm. Math. Phys. 1084 (1987), 535-589. MR0877637 (88g:53048)

[9] Klingenberg, W. Riemannian geometry. de Gruyter, Berlin (1982). MR0666697 (84j:53001)

[10] Kobayashi S. Transformation groups in differential geometry. Springer-Verlag, Berlin (1995). MR:1336823 (96c:53040)

[11] Kobayashi S. \& Nomizu, K. Foundations of differential geometry I. Interscience Publishers, N.Y. (1963). MR0152974 (27:2945)

[12] Foundations of differential geometry II. Interscience Publishers, N.Y. (1969). MR0238225 (38:6501)

[13] Lempert, L. \& Szőke, R. Global solutions of the homogeneous complex Monge-Ampère equation and complex structures on the tangent bundle of Riemannian manifolds. Math. Ann. 290 (1991), 689-712. MR1119947 (92m:32022)

[14] Marsden, J. \& Weisntein, A. Reduction of symplectic manifolds with symmetry. Rep. Math. Phys. 5 (1974), 121-130. MR0402819 (53:6633)

[15] O'Neill, B. The fundamental equations of a submersion. Mich. Math. J. 13 (1966), 459-469. MR0200865 (34:751)

Massachusetts Maritime Academy, Buzzards Bay, Massachusetts 02562

E-mail address: raguilar@maritime.edu 